\title{
\begin{tabular}{l|l} 
Mitraries & DSpace@MIT
\end{tabular}
}

\author{
MIT Open Access Articles
}

Stabilization of electron-scale turbulence by electron density gradient in national spherical torus experiment

The MIT Faculty has made this article openly available. Please share how this access benefits you. Your story matters.

Citation: Ruiz Ruiz, J., Y. Ren, W. Guttenfelder, A. E. White, S. M. Kaye, B. P. Leblanc, E. Mazzucato, et al. "Stabilization of Electron-Scale Turbulence by Electron Density Gradient in National Spherical Torus Experiment." Physics of Plasmas 22, no. 12 (December 2015): 122501.

As Published: http://dx.doi.org/10.1063/1.4936110

Publisher: American Institute of Physics (AIP)

Persistent URL: http://hdl.handle.net/1721.1/108751

Version: Final published version: final published article, as it appeared in a journal, conference proceedings, or other formally published context

Terms of Use: Article is made available in accordance with the publisher's policy and may be subject to US copyright law. Please refer to the publisher's site for terms of use. 


\section{Stabilization of electron-scale turbulence by electron density gradient in national}

spherical torus experiment

J. Ruiz Ruiz, Y. Ren, W. Guttenfelder, A. E. White, S. M. Kaye, B. P. Leblanc, E. Mazzucato, K. C. Lee, C. W. Domier, D. R. Smith, and H. Yuh

Citation: Physics of Plasmas 22, 122501 (2015); doi: 10.1063/1.4936110

View online: http://dx.doi.org/10.1063/1.4936110

View Table of Contents: http://aip.scitation.org/toc/php/22/12

Published by the American Institute of Physics 


\title{
Stabilization of electron-scale turbulence by electron density gradient in national spherical torus experiment
}

\author{
J. Ruiz Ruiz, ${ }^{1}$ Y. Ren, ${ }^{2}$ W. Guttenfelder,${ }^{2}$ A. E. White, ${ }^{1}$ S. M. Kaye, ${ }^{2}$ B. P. Leblanc, ${ }^{2}$ \\ E. Mazzucato, ${ }^{2}$ K. C. Lee, ${ }^{3}$ C. W. Domier, ${ }^{4}$ D. R. Smith, ${ }^{5}$ and H. Yuh ${ }^{6}$ \\ ${ }^{1}$ MIT-Plasma Science and Fusion Center, Cambridge, Massachusetts 02139, USA \\ ${ }^{2}$ Princeton Plasma Physics Laboratory, Princeton, New Jersey 08543, USA \\ ${ }^{3}$ National Fusion Research Institute, Daejeon, South Korea \\ ${ }^{4}$ University of California at Davis, Davis, California 95616, USA \\ ${ }^{5}$ University of Wisconsin-Madison, Madison, Wisconsin 53706, USA \\ ${ }^{6}$ Nova Photonics, Inc., Princeton, New Jersey 08540, USA
}

(Received 28 August 2015; accepted 6 November 2015; published online 4 December 2015)

Theory and experiments have shown that electron temperature gradient (ETG) turbulence on the electron gyro-scale, $k_{\perp} \rho_{e} \lesssim 1$, can be responsible for anomalous electron thermal transport in NSTX. Electron scale (high-k) turbulence is diagnosed in NSTX with a high-k microwave scattering system [D. R. Smith et al., Rev. Sci. Instrum. 79, 123501 (2008)]. Here we report on stabilization effects of the electron density gradient on electron-scale density fluctuations in a set of neutral beam injection heated $\mathrm{H}$-mode plasmas. We found that the absence of high-k density fluctuations from measurements is correlated with large equilibrium density gradient, which is shown to be consistent with linear stabilization of ETG modes due to the density gradient using the analytical ETG linear threshold in F. Jenko et al. [Phys. Plasmas 8, 4096 (2001)] and linear gyrokinetic simulations with GS2 [M. Kotschenreuther et al., Comput. Phys. Commun. 88, 128 (1995)]. We also found that the observed power of electron-scale turbulence (when it exists) is anti-correlated with the equilibrium density gradient, suggesting density gradient as a nonlinear stabilizing mechanism. Higher density gradients give rise to lower values of the plasma frame frequency, calculated based on the Doppler shift of the measured density fluctuations. Linear gyrokinetic simulations show that higher values of the electron density gradient reduce the value of the real frequency, in agreement with experimental observation. Nonlinear electron-scale gyrokinetic simulations show that high electron density gradient reduces electron heat flux and stiffness, and increases the ETG nonlinear threshold, consistent with experimental observations. (C) 2015 AIP Publishing LLC.

[http://dx.doi.org/10.1063/1.4936110]

\section{INTRODUCTION}

Particle and heat transport across the confining magnetic field of present day magnetic confinement fusion devices exceed neoclassical collisional diffusion predictions by several orders of magnitude. Of particular importance for a tokamak fusion reactor is the transport of electron energy, since a large fraction of the energy from fusion products would be deposited to the electrons. In most present day tokamaks, electron thermal transport is consistently found anomalous in a vast range of operating regimes. Understanding electron thermal transport and the causes giving rise to the anomalous levels experimentally observed is a key requirement in order to achieve a predictive capability for the confinement of future fusion devices such as ITER or FNSF (Fusion Nuclear Science Facility). ${ }^{4}$

Ample theory and numerical simulations ${ }^{5-12}$ have suggested that micro instabilities driven by the electron temperature gradient (ETG) could be responsible for anomalous electron thermal transport levels observed experimentally. The ETG toroidal instability has a characteristic scale length on the order of the electron gyro radius $\left(k_{\perp} \rho_{e} \leq 1\right)$. Ion thermal transport has been consistently observed at neoclassical levels in typical NSTX H-mode plasmas; ${ }^{13,22}$ however, anomalous levels of electron thermal transport make it the dominant thermal loss channel in most operating regimes.

Several mechanisms have recently been reported to affect ETG turbulence. The $E \times B$ shear has been observed to reduce electron scale turbulence (measured with a high-k scattering system $^{1,14,15}$ ). Negative magnetic shear configurations can also affect ETG turbulence through the formation of internal transport barriers as reported in Refs. 16 and 17. The influence of electron density gradient on ETG turbulence was first pointed out by Ren et al. in Ref. 18, where the normalized local electron density gradient $\left(R_{0} / L_{n_{e}}\right)$ was reported to stabilize longer wavelength modes $\left(k_{\perp} \rho_{s} \lessgtr 10\right)$ and reduced the plasma effective thermal diffusivity. Nonlinear gyrokinetic simulations carried out for the same plasma discharge and reported in Ref. 19 showed in fact that the fractional electron heat flux $Q_{e}$ was greatly reduced at lower ETG wavenumbers and at the same time shifted towards higher wavenumbers.

Linear gyrokinetic simulations are able to capture the ETG instability dependence on the electron density gradient. The threshold for the ETG instability can be quantified by a critical gradient. An analytical expression for the ETG critical gradient was derived in Ref. 2 by Jenko et al. by carrying out many linear gyrokinetic simulations. The critical gradient is given by 


$$
\left(R_{0} / L_{T_{e}}\right)_{c r i t}=\max \left\{\begin{array}{c}
0.8 R_{0} / L_{n_{e}} \\
(1+\tau)(1.33+1.99 \hat{s} / q)(1-1.5 \epsilon)(1+0.3 \epsilon d \kappa / d \epsilon)
\end{array}\right\},
$$

where $\tau=Z_{\text {eff }} T_{e} / T_{i}$. In this expression, $R_{0} / L_{X}$ are normalized gradients of the quantity $X$, such that $R_{0} / L_{X}=-R_{0} /(\mathrm{d} \ln \mathrm{X} / d r)$, and $R_{0}$ is the corresponding flux surface center. $Z_{\text {eff }}$ is the effective ion charge, $T_{e}$ is the electron temperature, $T_{i}$ is the ion temperature, and $\hat{s}$ and $q$ are the magnetic shear and safety factor, respectively. $\epsilon$ is the inverse aspect ratio and $\kappa$ is the elongation. According to expression (1), the electron density gradient is predicted to have a stabilizing effect on the ETG instability. High values of the electron density gradient will make the $0.8 R_{0} / L_{n_{e}}$ term dominant in expression (1); thus, it will determine the value of the critical gradient which should have a stabilizing effect on the ETG instability.

In Section II we present the experimental setup and analysis of the electron density gradient effect on high-k electron density fluctuation spectra from the high-k scattering system. We observe that large equilibrium density gradient is able to suppress the observed high-k density fluctuations, consistent with linear stabilization of ETG modes by using the analytical ETG linear threshold ${ }^{2}$ as was discussed in Refs. 18 and 19. In this work, we also observe that the scattered power from electron-scale turbulence is anti-correlated with the equilibrium density gradient, suggesting density gradient as a nonlinear stabilizing mechanism of high-k turbulence. In Section III we show the impact of the electron density gradient on linear growth rates and real frequency using linear gyrokinetic simulations with the gyrokinetic code GS2. ${ }^{3}$ A clear correlation is observed between the wavenumber value at the maximum linear growth rate and the local value of the electron density gradient. Higher values of the electron density gradient are also shown to reduce the value of the real frequency of instability from GS2. Experimental plasma frame frequencies of fluctuations exhibit the same dependence with density gradient, reinforcing the frequency dependence on density gradient. In Section IV we present preliminary results from nonlinear gyrokinetic simulations using the code GYRO. ${ }^{32}$ These simulations show how high electron density gradient reduces the electron heat flux and stiffness, and increases the ETG nonlinear threshold, reaffirming a nonlinear change in ETG turbulence and fluctuation spectrum from large equilibrium density gradient as predicted from previous ETG nonlinear simulations. ${ }^{19,34}$

\section{EXPERIMENTAL STUDY OF ELECTRON DENSITY GRADIENT DEPENDENCE}

Electron density fluctuations on the electron gyro radius scale $\left(k_{\perp} \rho_{e} \lesssim 1\right)$ were measured with a $280 \mathrm{GHz}$ microwave coherent scattering system (the high-k scattering system) implemented on NSTX. ${ }^{1}$ The scattering system consists of five collection channels that simultaneously measure five different wavenumbers in the range $5 \leqq k_{\perp} \lesssim 30 \mathrm{~cm}^{-1}$. Heterodyne receivers installed on each channel allow to determine the direction of propagation of the observed fluctuations. The wavenumber resolution of the observed electron density fluctuations is $\Delta k \approx \pm 0.7 \mathrm{~cm}^{-1}$ and the radial resolution $\Delta R \approx \pm 2 \mathrm{~cm}$ (details about the wavenumber and radial resolution of the high-k system can be found in Refs. $20,21)$. Measured wave vectors are primarily radial $k_{r}$, with a small binormal component $k_{b}$ satisfying $k_{b} / k_{r} \approx 0.2-0.3\left(k_{r}\right.$ is the radial component perpendicular to the flux surface and $k_{b}$ is the binormal component perpendicular to the local magnetic field but inside the flux surface). The near mid-plane trajectory of the probe beam and the k-response are computed using a ray tracing code. In Fig. 1 is shown the trajectory of 4 channels of the high-k scattering system for NSTX shot 141767. In the experiment we present here, the scattering system is sensitive to fluctuations taking place at $R \approx 135 \mathrm{~cm}$ (major radius of NSTX is $0.85 \mathrm{~m}$, minor radius $0.68 \mathrm{~m}$, and $\mathrm{r} / \mathrm{a} \sim 0.7)$. Channel 1 measures $k_{r} \rho_{s} \sim 13-17$ and $k_{b} \rho_{s} \sim 3-4$, which in physical units typically corresponds to $k_{r} \sim 18-25 \mathrm{~cm}^{-1}$ and $k_{b} \sim 5 \mathrm{~cm}^{-1}$ ( $\rho_{s}$ is computed using local values of electron temperature $T_{e}$ and magnetic field from LR circuit model with Data Fitting capabilities (LRDFIT) equilibrium reconstruction). The electron and ion gyro-radii typically have values $\rho_{e} \approx 0.1 \mathrm{~mm}$ and $\rho_{i} \approx \rho_{s} \approx 0.7 \mathrm{~cm}$ in these NSTX plasmas.

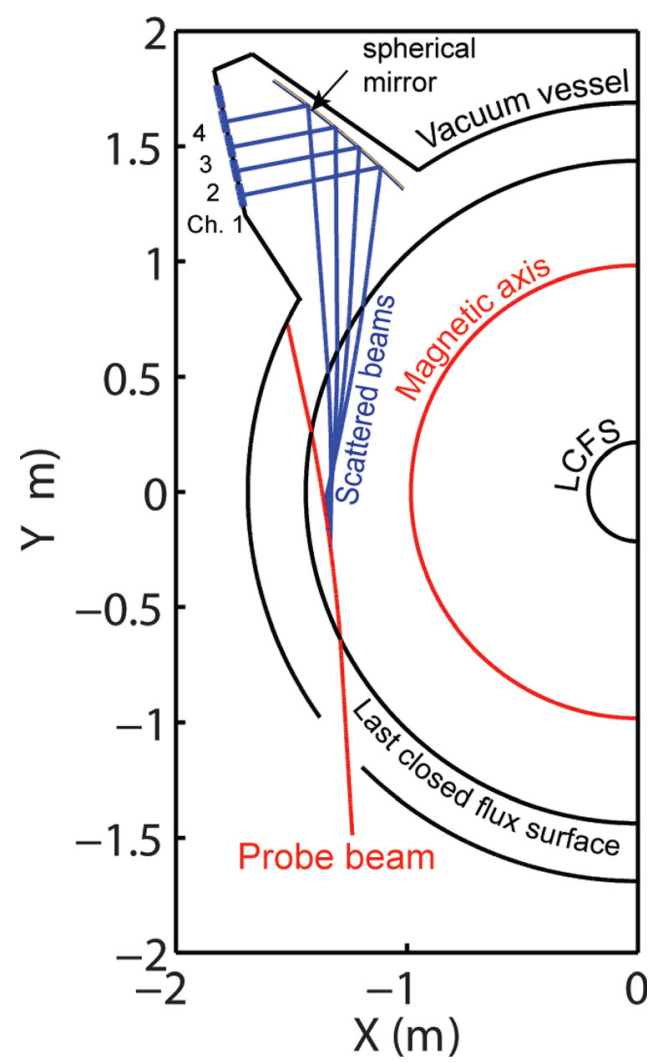

FIG. 1. (a) Experimental configuration of high-k scattering experiment for shot 141767 (view from the top). Probe beam is launched nearly on the midplane. Gaussian beam propagation is calculated using a ray-tracing code. 


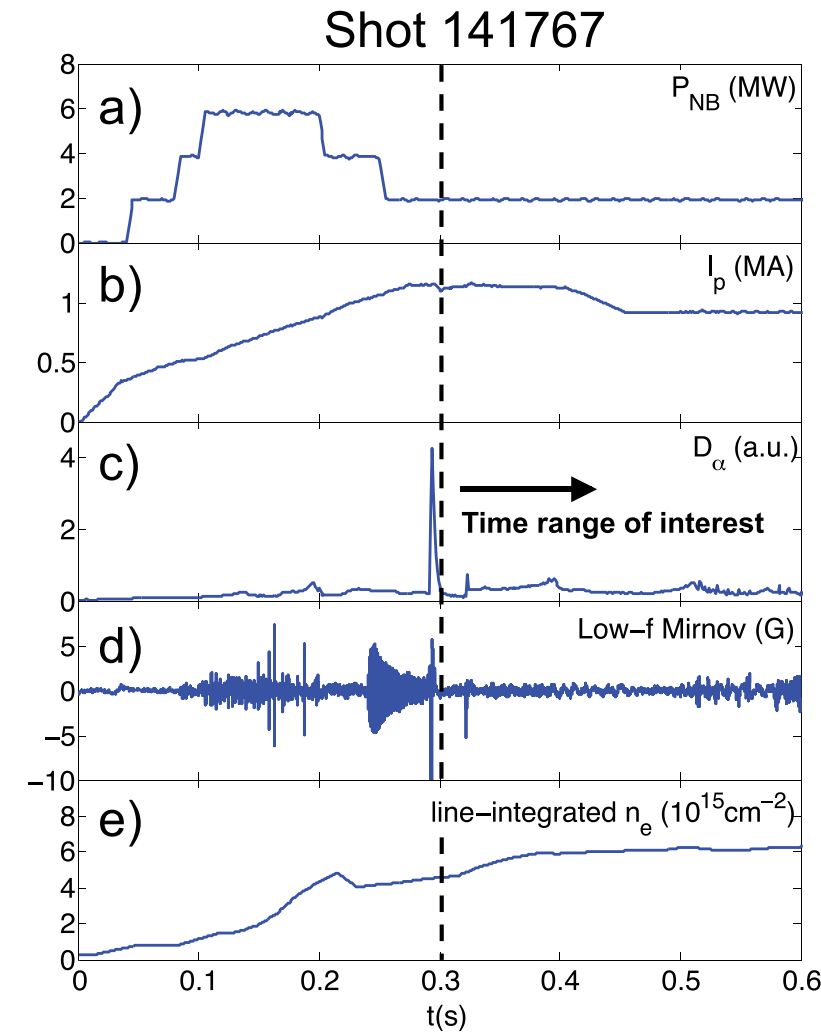

FIG. 2. An overview of shot 141767. From top to bottom: NBI heating power $P_{N B}$ (MW), plasma current $I_{p}$ (MA), $D_{\alpha}$ emission (not calibrated), low frequency magnetic fluctuations $(\mathrm{G})$, and line-integrated density $\bar{n}_{e}\left(10^{15} \mathrm{~cm}^{-2}\right)$. High Harmonic Fast Wave (HHFW) heating was absent during this discharge.

Here we focus on a particular experiment of an NSTX neutral beam injection (NBI)-heated H-mode discharge (shot 141767), featuring a controlled plasma current ramp-down at relatively constant line-integrated electron density (Fig. 2(e)) and toroidal magnetic field $\left(B_{t} \sim 4.6-5 \mathrm{kG}\right)$. The time preceding $0.3 \mathrm{~s}$ shows high MHD activity from the low-frequency (low-f) Mirnov signal. High MHD activity will contaminate the scattering system fluctuation measurements, thus we will only focus on the time range after $\mathrm{t}=0.3 \mathrm{~s}$ where MHD activity is observed to be quite low.

In Figure 3(a) are shown the electron density fluctuation spectrograms for 3 channels. Stray radiation is present as $f=0$ central peaks in Figs. 3(a) and 3(b). The spectral features at negative frequency after $t=0.3 \mathrm{~s}$ correspond to high- $\mathrm{k}$ density fluctuations. Further analysis presented here will focus on channel 1. In Figure 3(b) are shown time slices of channel 1 corresponding to the frequency spectrum of electron density fluctuations.

In Figure 4 is plotted the total scattered power from channel 1 (integrated spectrogram in frequency from Fig. 3 (a)) along with the experimental and critical electron temperature gradient at the scattering location (black and red, respectively, in Fig. 4(b)), and the difference between the two in Fig. 4(c). The local values of electron density $n_{e}$ and electron temperature $T_{e}$ present in the critical ETG formula are measured with the Multi Point Thomson Scattering diagnostic at NSTX $\left(\mathrm{MPTS}^{26}\right)$. Ion temperature $T_{i}$ and effective ion charge $Z_{\text {eff }}$ are measured with the Charge Exchange Recombination Spectroscopy system $\left(\right.$ CHERS $\left.^{27}\right)$. Magnetic shear $\hat{s}$, safety factor $q$, and inverse aspect ratio $\epsilon$ are obtained using LR circuit model with Data Fitting capabilities $^{28}$ equilibrium reconstructions constrained by magnetic pitch angle measurements from the Motional Stark Effect (MSE) diagnostic. ${ }^{29}$

For early times $(t \lesssim 0.33 \mathrm{~s})$, the experimental temperature gradient is at marginal stability levels with respect to the critical gradient, and no high-k fluctuations are observed at that time in Fig. 4(a). Note that the high spike at $t \sim 292 \mathrm{~ms}$ from Fig. 4(a) corresponds to a powerful ELM (Edge Localized Mode) event and does not correspond to ETG fluctuations. As the critical gradient suddenly decreases at $t \sim 330 \mathrm{~ms}, R / L_{T_{e}}^{\text {exp }}-\left(R / L_{T_{e}}\right)_{c r i t}$ increases in Fig. 4(c), and ETG becomes unstable. At that time, electron density fluctuations start to develop in Fig. 4(a). The critical gradient formula (Eq. (1)) is shown to correctly predict the stability of ETG modes. Note how $R / L_{T_{e}}^{e x p}-\left(R / L_{T_{e}}\right)_{\text {crit }}$ remains positive and fluctuations persist until the end of the discharge.

The impact of density gradient on the linear stabilization of ETG modes is clearly observed in Fig. 5. The electron density gradient term $\left(0.8 R_{0} / L_{n_{e}}\right.$, blue curve in Fig. $\left.5(\mathrm{c})\right)$ is dominant for most part of the time range of interest ( $\mathrm{t}>0.3 \mathrm{~s}$ ). High values of $R_{0} / L_{n_{e}}$ for times $t \lesssim 330 \mathrm{~ms}$ are responsible for raising the ETG critical gradient resulting in marginally stable ETG modes, and no high-k density fluctuations are observed in Figs. 5(a) and 5(b). A sudden drop of $R_{0} / L_{n_{e}}$ after $t \approx 330 \mathrm{~ms}$ produces the drop of the ETG critical gradient (cf. Fig. 4(b)), making ETG modes unstable, and density fluctuations appear in Figs. 4(a), 5(a), and 5(b). This
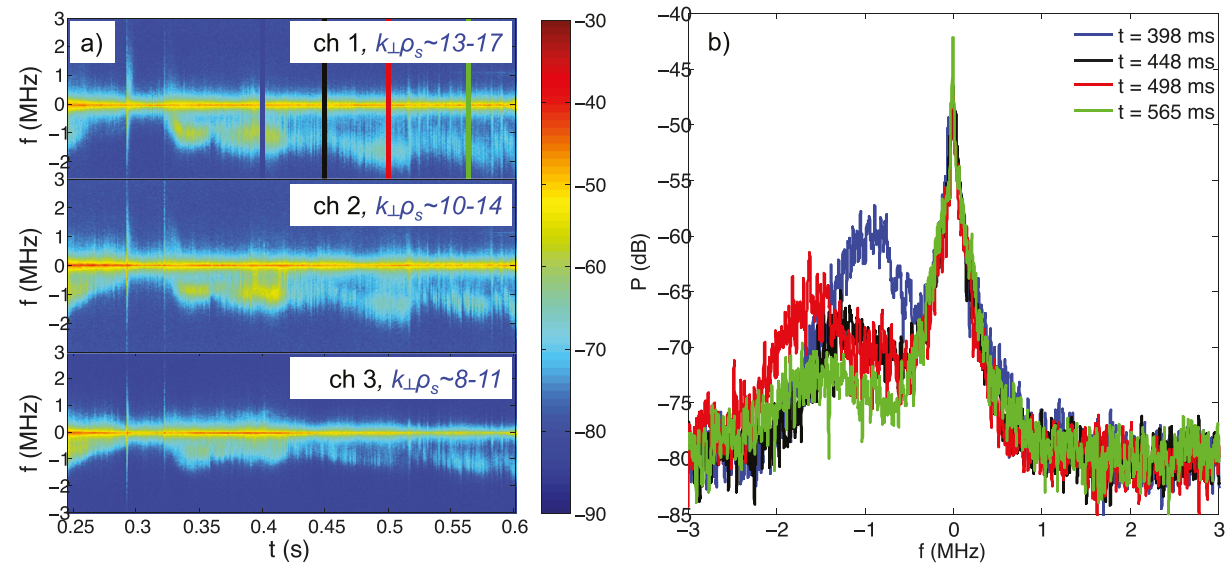

FIG. 3. (a) Spectrogram of high-k density fluctuations from channels 1,2 , and 3 of shot 141767 of the high-k scattering system at NSTX. (b) Frequency spectrum of fluctuations corresponding to times as shown on (a) by vertical color lines from channel 1. Note (b) are simply time slices of channel 1 at the vertical color lines. Each channel is sensitive to a different wavenumber $k_{\perp} \rho_{s}$ as is indicated in (a). 


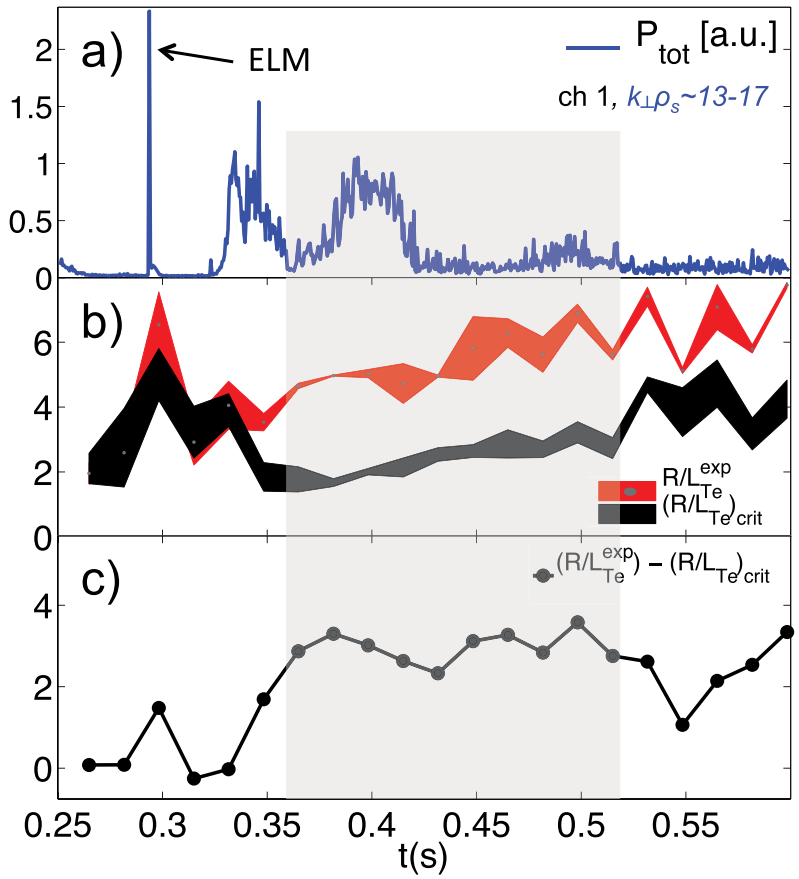

FIG. 4. (a) Total scattered power from channel 1 of the high-k scattering system. The total scattered power is found integrating the frequency spectrogram in Fig. 3(a) in frequency. (b) Experimental (red) and critical temperature gradient (black) at the scattering location. (c) The difference $R / L_{T_{e}}^{\text {exp }}-\left(R / L_{T_{e}}\right)_{\text {crit }}$ indicates whether ETG is unstable or not, and should correlate to the presence of electron density fluctuations.

confirms the linear stabilization of ETG modes due to density gradient by using the ETG critical gradient (Eq. (1)).

During the time span $t \sim 0.36-0.53 \mathrm{~s}$ (grey time panel in Fig. $4(\mathrm{c})), R / L_{T_{e}}^{\exp }-\left(R / L_{T_{e}}\right)_{\text {crit }}$ has a very similar value. However, this very similar value of $R / L_{T_{e}}^{e x p}-\left(R / L_{T_{e}}\right)_{\text {crit }}$ gives rise to a very different fluctuation amplitude in Fig. 4(a). In addition, the electron density gradient term becomes subdominant between $360 \lesssim t \lesssim 410 \mathrm{~ms}$ (blue curve in Fig. 5(c)), and an enhancement of high-k fluctuations is observed in Fig. 3(b) (blue curve at $\mathrm{t}=398 \mathrm{~ms}$ ) as well as in the total scattered power in Figs. 5(a) and 5(b). This suggests a nonlinear dependence of the fluctuation amplitude with density gradient, in addition to the linear threshold dependence in $R / L_{T_{e}}^{e x p}-\left(R / L_{T_{e}}\right)_{\text {crit }}$.

After a sudden increase in the electron density gradient at $t \approx 520 \mathrm{~ms}$, high-k fluctuations appear to mitigate (see green curve in Fig. 3(b) and Figs. 5(a) and 5(b)). This reduction could be due to a linear stabilizing effect (reduction of $\left.R / L_{T_{e}}^{\text {exp }}-\left(R / L_{T_{e}}\right)_{c r i t}\right)$ due to density gradient, but density gradient might also be acting as a nonlinear stabilizing mechanism at $t \gtrsim 520 \mathrm{~ms}$. In any event, the anti-correlation shown between the density gradient and the fluctuation amplitude remains clear.

The current ramp-down from $t=400$ and $t=450 \mathrm{~ms}$ does not appear to have a direct impact on the ETG critical gradient, since the $\hat{s} / q$ term remains subdominant during the current ramp-down phase (Fig. 5(c)). In Fig. 5(c) is also plotted the ETG critical gradient explicitly computed with GS2 (purple triangles). Notice the fair agreement between the GS2 calculated critical gradient and the critical gradient formula (black curve in Fig. 5(c)) despite the high- $\beta$, tight aspect ratio condition of NSTX. The critical gradient formula developed by Jenko et al.

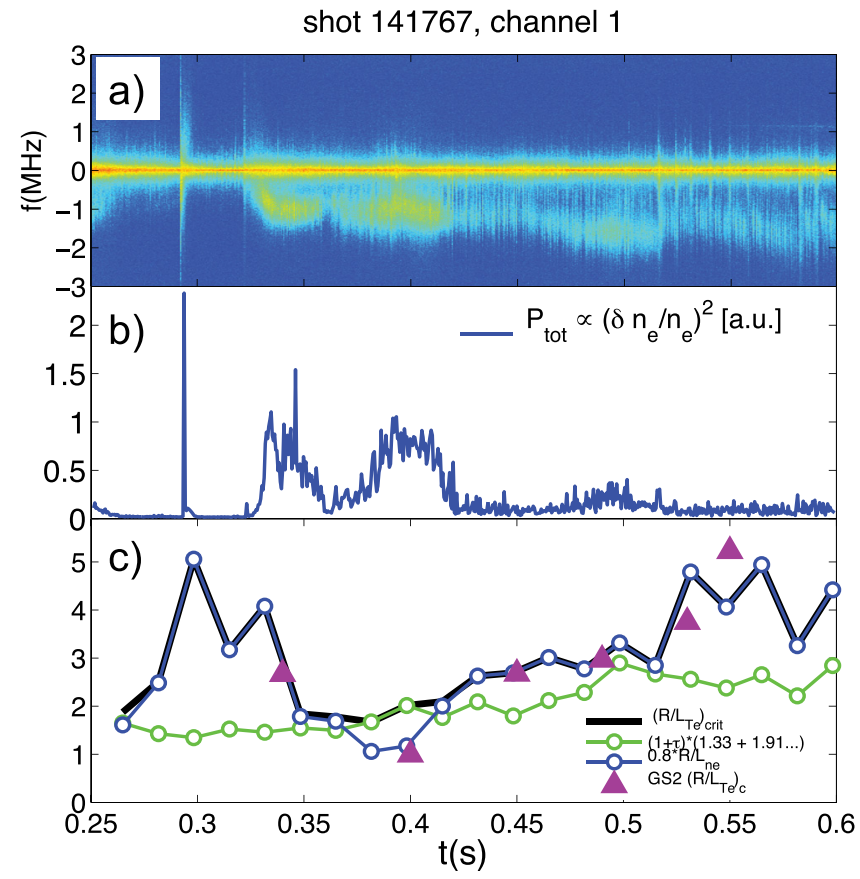

FIG. 5. (a) Fluctuation spectrogram from channel 1. (b) Total scattered power from channel 1 of the high-k scattering system. (c) Critical gradient formula (black) and both terms in the max function (green and blue curves). Triangles indicate the critical gradient computed explicitly using GS2.

in Ref. 2 was previously observed to have fairly good agreement with explicitly calculated critical gradient using linear gyrokinetic simulations in NSTX plasmas. ${ }^{25}$ This supports our previous comparisons between the experimental temperature gradient and the critical gradient formula, and we will assume that the critical gradient formula is correct.

The frequencies detected by the high-k system in the lab frame $\left(f_{\text {lab }}\right)$ are Doppler shifted from intrinsic frequencies of measured fluctuations in the plasma frame $f_{p}$, by a quantity $f_{D} \approx k_{t} v_{t} / 2 \pi$, where $f_{p}=f_{\text {lab }}-f_{D}$ (Fig. 6(a)). Here $k_{t}$ is the toroidal component of the fluctuation wavenumber (obtained from a ray-tracing code), and $v_{t}$ is the $E \times B$ component of the toroidal rotation velocity at the scattering location, obtained from CHERS measurements ${ }^{27}$ and numerical transport code calculations. The quantity $f_{\text {lab }}$ is defined as $\int f S(f) d f / \int S(f) d f$, and is shown in Figure 6(a) for the time $t=398 \mathrm{~ms}$, as a black line passing roughly through the maximum of the spectral peak of fluctuations at $f \approx-1000 \mathrm{kHz}$. $S(f)$ is the frequency spectral power in Figure 6(a) at $t=398 \mathrm{~ms}$.

At each time in the analysis, the Doppler-subtracted plasma frame frequency of fluctuations $f_{p}$ can be plotted against the experimental perpendicular wavenumber (obtained by a ray-tracing code). This provides an experimental dispersion relation of the measured fluctuations, and can be compared with a dispersion relation obtained from a linear gyrokinetic simulation. In Figure 6(b), the plasma frame frequency $f_{p}$ is plotted against the perpendicular wavenumber $k_{\perp}$ of the measured fluctuation by the high-k scattering system. In this notation $k_{\perp}=\sqrt{k_{b}^{2}+k_{r}^{2}}$, where $k_{b}$ is the binormal component of the measured wavenumber and $k_{r}$ is the radial component. At a given time, the different wavenumbers correspond to the different channels of the high-k scattering diagnostic. 

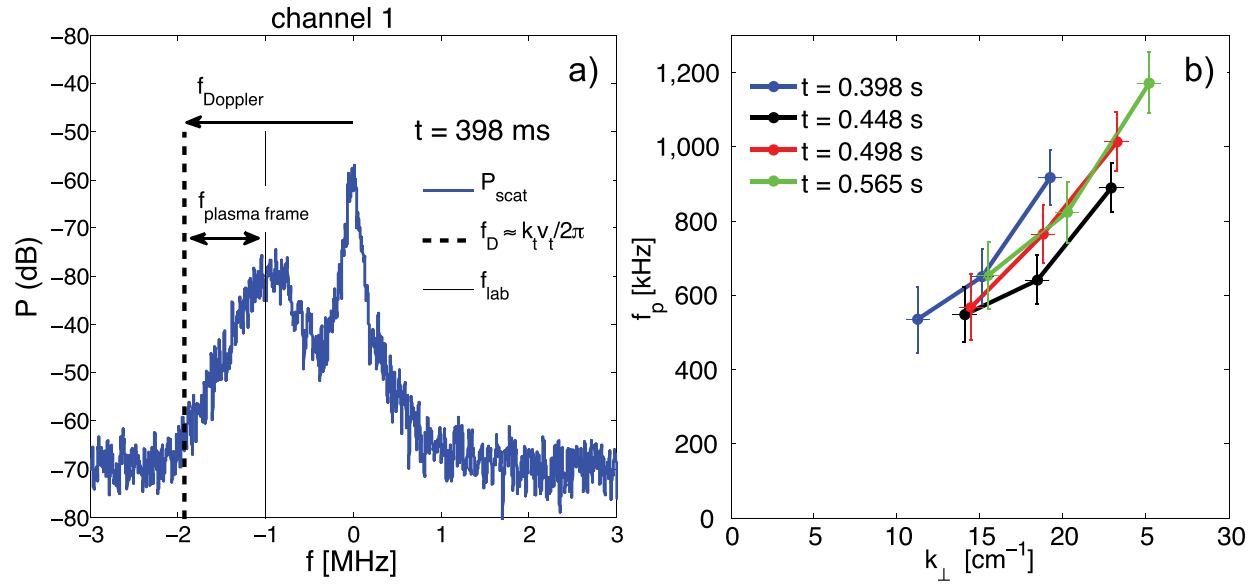

FIG. 6. (a) Scattered power from channel 1 of high-k scattering diagnostic, at time $\mathrm{t}=398 \mathrm{~ms}$. Fluctuation frequencies are Doppler shifted by $f_{D} \approx k_{t} v_{t} /$ $2 \pi$. The plasma frame frequency of fluctuations $f_{p}$ is obtained by $f_{p}=f_{\text {lab }}$ $-f_{D}$. (b) Dispersion relation obtained experimentally from measured electron density fluctuations.

The experimental dispersion relation shown in Fig. 6 is plotted at four different times. We note that the uncertainties in $f_{p}$ come from $k_{t}$ and $v_{t}$. Higher wavenumbers give rise to higher plasma frame frequencies. In Section IV, we wish to compare the plasma frame real frequency with real frequencies calculated by GS2 linear analysis.

A wavenumber spectrum of fluctuations can be obtained by integrating the frequency spectrum (Fig. 3(a)) from the different channels. In Figure 7, the wavenumber values are calculated using a ray-tracing code. At $t=398 \mathrm{~ms}$ the fluctuation level is high, and corresponds to a time when the electron density gradient term (Fig. 5(c)) is subdominant in the critical gradient formula. At $t=448 \mathrm{~ms}$ the fluctuation level at low and mid wavenumbers $\left(k_{\perp} \rho_{s} \lesssim 12\right)$ has been greatly reduced. At this time, the electron density gradient term has become dominant in the critical gradient. At $t=498 \mathrm{~ms}$ the electron density gradient term is still dominant (marginally dominant) in the critical ETG. At the same time, lower-k $\left(k_{\perp} \rho_{s} \sim 10\right)$ density fluctuations have no significant change with respect to $t=448 \mathrm{~ms}$, but higher-k $\left(k_{\perp} \rho_{s} \gtrsim 12\right)$ density fluctuations at $t=498 \mathrm{~ms}$ have noticeably increased from $t=448 \mathrm{~ms}$ levels. At $t=565 \mathrm{~ms}$, the fluctuation wavenumber spectrum is similar to the spectrum at $t=498 \mathrm{~ms}$ at higher-k wavenumbers $\left(k_{\perp} \rho_{s} \gtrsim 13-14\right)$ but the lower-k fluctuation level has also increased with respect to $t=498 \mathrm{~ms}$. It has

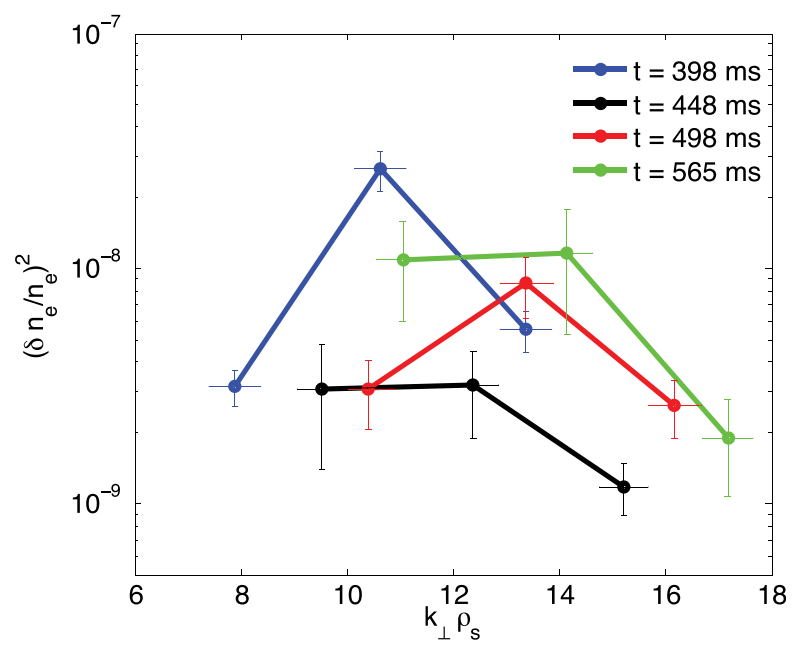

FIG. 7. Wavenumber spectrum of electron density fluctuations. Note $k_{\perp}=\sqrt{k_{b}^{2}+k_{r}^{2}}$. been previously observed how the electron density gradient is most efficient in stabilizing lower- $k$ ETG fluctuations $\left(k_{\perp} \rho_{s} \lesssim 10\right)$ and at the same time shifting the spectrum towards higher k. ${ }^{18}$ This is indeed what we observe in Fig. 7 for times $t=398,448$, and $498 \mathrm{~ms}$. However, at $t=565 \mathrm{~ms}$ (highest density gradient, cf. blue curve in Fig. 5(c)) density fluctuations for $k_{\perp} \rho_{s} \approx 11$ (lower- $k$ ) increase with respect to $t=498 \mathrm{~ms}$ levels, contradicting this last statement. We note that the shift of wavenumbers to higher-k might also be due to the fact that lower wavenumbers become inaccessible at higher density gradients due to beam refraction effects.

In Fig. 8 we present a compact way to quantify the correlations previously found. At each time in the analysis (time span $0.348<t<0.565 \mathrm{~s}$ ), we calculate maximum fluctuation level $\left(\delta n_{e} / n_{e}\right)_{\text {max }}^{2}$ and the wavenumber corresponding to the maximum fluctuation level $k_{\perp} \rho_{s}\left(\left(\delta n_{e} / n_{e}\right)_{\max }^{2}\right)$. The local value of the electron density gradient is also calculated, and one can plot $\left(\delta n_{e} / n_{e}\right)_{\text {max }}^{2}$ and $k_{\perp} \rho_{s}\left(\left(\delta n_{e} / n_{e}\right)_{\text {max }}^{2}\right)$ as a function of the electron density gradient, as is shown in Figs. 8(c) and $8(\mathrm{~d})$. At the particular time of $t=0.432 \mathrm{~s}$, these values are taken from the orange dot in Fig. 8(a) and mapped in Figs. $8(\mathrm{c})$ and $8(\mathrm{~d})$. The slight correlation observed between $\left(\delta n_{e} / n_{e}\right)_{\text {max }}^{2}$ and density gradient can suggest that higher density gradient may lead to lower fluctuation amplitude (as already suggested in Fig. 5). A clear correlation is found between $k_{\perp} \rho_{s}\left(\left(\delta n_{e} / n_{e}\right)_{\text {max }}^{2}\right)$ and density gradient. We discuss the validity of this correlation in the last paragraph of this section.

To exploit the correlation between the plasma frame frequency of fluctuations and density gradient, we pick a fixed wavenumber, and calculate at each time, the Dopplersubtracted plasma frame frequency corresponding to that fixed wavenumber (as shown in Fig. 6). We choose the wavenumber $k_{\perp} \rho_{s}=13.2$, and the plasma frame frequency of fluctuations corresponding to that wavenumber for $t=0.432 \mathrm{~s}$ is shown in Fig. 8(b) as a red dot. In a similar way as was done with the fluctuation levels, $\omega_{p}\left(k_{\perp} \rho_{s}=13.2\right)$ is plotted against the local value of $R_{0} / L_{n_{e}}$ at the corresponding time in Fig. 8(e) (see red dot corresponding to a time $t=0.432 \mathrm{~s}$ ). A noticeable correlation is observed with a correlation coefficient of $R^{2} \approx 47 \%$. This suggests that density gradient is affecting the observed fluctuations by decreasing the plasma frame frequency of fluctuations. 

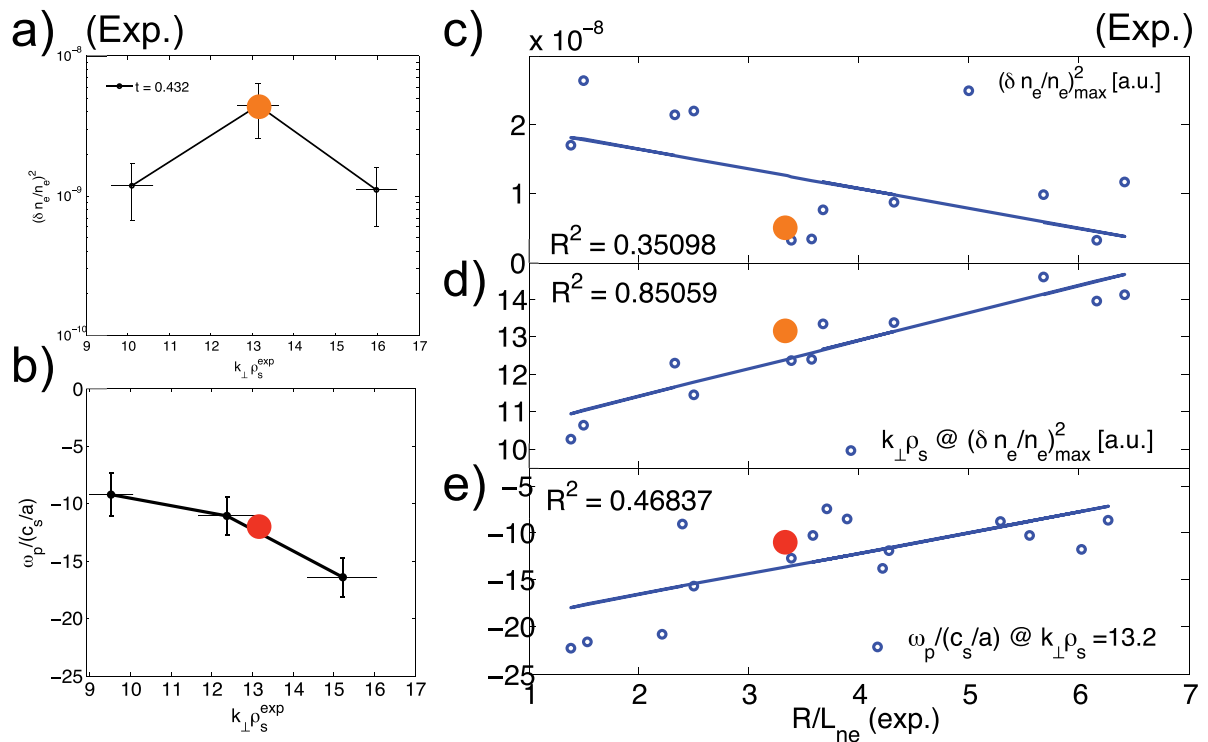

FIG. 8. This figure is the experiment analog of Figure 11. At each time, the maximum fluctuation level $\left(\delta n_{e} / n_{e}\right)^{2}$ and the wavenumber corresponding to the maximum fluctuation level $k_{b} \rho_{s} @\left(\delta n_{e} / n_{e}\right)_{\max }^{2}$ are recorded (a), and are plotted against the local value of the density gradient in (c) and (d). The real frequency is calculated at a fixed wavenumber $\left(k_{\perp} \rho_{s}=13.2\right)$ and is plotted in (e) as a function of density gradient.

The correlation found between the wavenumber at maximum fluctuation level and density gradient in Fig. 8(d) can have a tricky interpretation. Concerning the experiment, it is true that a gradient in the electron density affects the measurement wavenumber from the different channels by refracting the incident beam of radiation. The measurement wavenumber from each channel slightly changes in time, and in fact, if one were to plot the measured wavenumber from a particular channel with respect to the density gradient, one would find a very clear correlation. Refraction acts to increase the detected wavenumber by each channel of the scattering diagnostic. This effect is independent of plasma turbulence levels and is purely a refraction effect due to density gradient. Following this argument, one could interpret Fig. 8(d) as being purely a refraction effect. However, Fig. 8(d) is picking the wavenumber that gives rise to a maximum fluctuation level, which itself is a turbulence-related quantity. Even though a density gradient is indeed bending the probe beam and resulting in bigger detected wavenumbers, the fact that the scattering system is detecting a maximum fluctuation amplitude for a midwavenumber (a case with a clear maximum in $\left.\left(\delta n_{e} / n_{e}\right)^{2}\right)$ and the fact that this mid wavenumber is correlated with the density gradient could indeed suggest that the density gradient is shifting wavenumber of the turbulence present to even higher values. However, beam refraction effects are present in our experiment, and we are at this point unable to decouple the effects of beam refraction on the probe beam due to density gradient from a true turbulence effect of density gradient on the wavenumber of the turbulence present. We should mention however that linear and nonlinear simulations do suggest correlations between density gradient and the wavenumber at the maximum growth rate and fractional electron heat flux, respectively, and will be discussed in Sections III-V.

\section{GYROKINETIC SIMULATION STUDY OF DENSITY GRADIENT DEPENDENCE}

In this section we perform a gyrokinetic simulation study of the corresponding NSTX plasma discharge presented in Section II. Linear gyrokinetic simulations were carried out with the gyrokinetic code GS2. ${ }^{3}$ Preliminary nonlinear gyrokinetic simulations were carried out with the gyrokinetic code GYRO. ${ }^{32}$

\section{A. Linear stability analysis}

We report on the influence of the electron density gradient on high-k linear growth rates and real frequencies corresponding to linearly unstable wavenumbers from the GS2 gyrokinetic code. ${ }^{3}$ GS2 is an initial value gyrokinetic code that uses flux tube geometry. In its linear version, GS2 tracks the fastest growing modes for a given pair of radial and poloidal wavenumbers. In the linear simulations presented, the radial component $k_{r}$ was set to 0 to find the most unstable mode. Linear growth rates and real frequencies are plotted against $k_{\theta}$. Electromagnetic effects and collisions were also included in these linear runs. The linear simulations shown in this section use local Miller equilibrium. ${ }^{30}$

Linear stability analysis was carried out for low-k $\left(k_{\perp} \rho_{s}\right.$ $\lesssim 1)$ and high-k $\left(k_{\perp} \rho_{s} \gg 1\right)$ wavenumber values. The wavenumber $k_{\theta}$ is normalized with respect to the ion sound gyro radius $\rho_{s}$, and the computed real frequencies and linear growth rates are normalized by the quantity $c_{s} / a$, where $c_{s}$ is the ion sound speed and $a$ is the minor radius.

The computed real frequencies and linear growth rates are shown in Figs. 9(a) and 9(b) for times $t=0.398,0.448$, 0.498 , and $0.565 \mathrm{~s}$ corresponding to Thomson scattering time points (MPTS). GS2 uses experimental profiles at the closest experimental time points, and electron density and temperature values at those times were used as input for linear gyrokinetic calculations in Fig. 9. Low-k linear simulations on the ion-scale were carried out for wavenumbers $0.1 \lesssim k_{\theta} \rho_{s}$ $\lesssim 1$ and high-k simulations for wavenumbers $10 \leqq k_{\theta} \rho_{s} \lesssim$ 60 .

Figure 9(a) shows real frequencies $\omega_{r}$ calculated by GS2. The instability propagates in the electron diamagnetic drift direction at high-k and is consistent with the toroidal ETG instability $\left(\omega_{r}<0\right.$ in GS2 corresponds to electron 

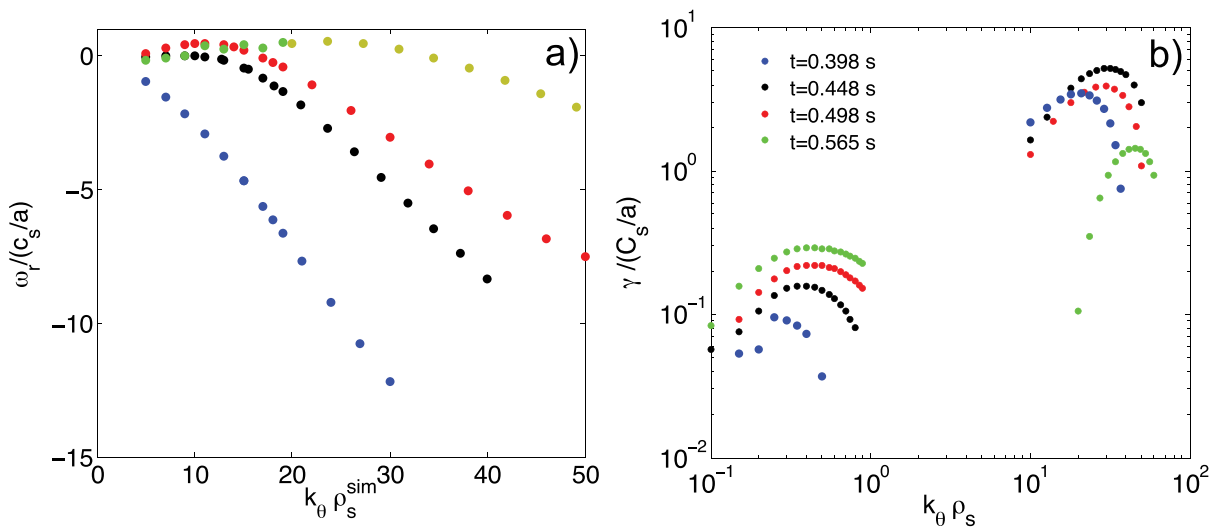

FIG. 9. (a) Real frequency of instability $\omega_{r}$. Notice $\omega_{r}<0$ indicates propagation in the electron diamagnetic drift direction in GS2. (b) Linear growth rates computed with GS2 for low-k $\left(0.1 \lesssim k_{\theta} \rho_{s} \lesssim 1\right)$ and high-k wavenumbers $\left(10 \leqq k_{\theta} \rho_{s} \lesssim 60\right)$. Notice the linearly unstable high-k wavenumbers are shifting to even higher wavenumbers as time progresses.

diamagnetic drift direction). Notice from Figs. 5(c) and 14(e) that the local electron density gradient is increasing in time between times $t=398 \mathrm{~ms}$ and $t=565 \mathrm{~ms}$ and we can establish a correlation between the local value of the electron density gradient and the real frequency from GS2. The strong variation of $\omega_{r}$ in time can be explained as an electron density gradient effect. Figure 9(b) shows that low-k linear growth rates monotonically increase in time throughout the shot and are substantially lower than high-k linear growth rates (note the log-scale on the $\mathrm{x}$-axis used to represent both low-k and high-k modes). High-k linear growth rates tend to increase for $t<0.448 \mathrm{~s}$ and they decrease afterwards. The linearly unstable high-k wavenumbers shift to even higher wavenumbers as time progresses, which can be justified by an increasing value of the local electron density gradient (Figs. 5(c) and 14(e)).

A scan of the electron density gradient is performed about its experimental value, keeping all other experimental parameters constant. This scan is carried out at two different times in the discharge $(t=398,565 \mathrm{~ms})$. Figure 10 shows real frequencies and linear growth rates computed for different values of the electron density gradient, and at two different times. At $t=565 \mathrm{~ms}$, the electron density gradient has a high value (cf. blue term in Figs. 5(c) and 14(e)) and is the dominant term in the critical gradient formula (Eq. (1)). A scan on the local $R_{0} / L_{n_{e}}$ shows a big effect on the real
(Sim.)
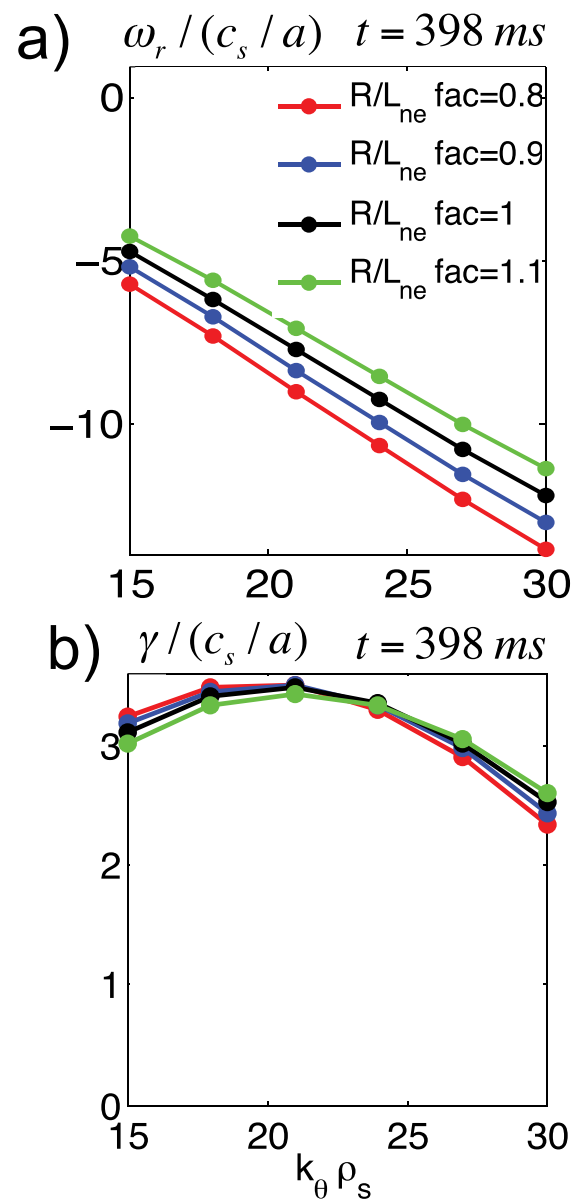
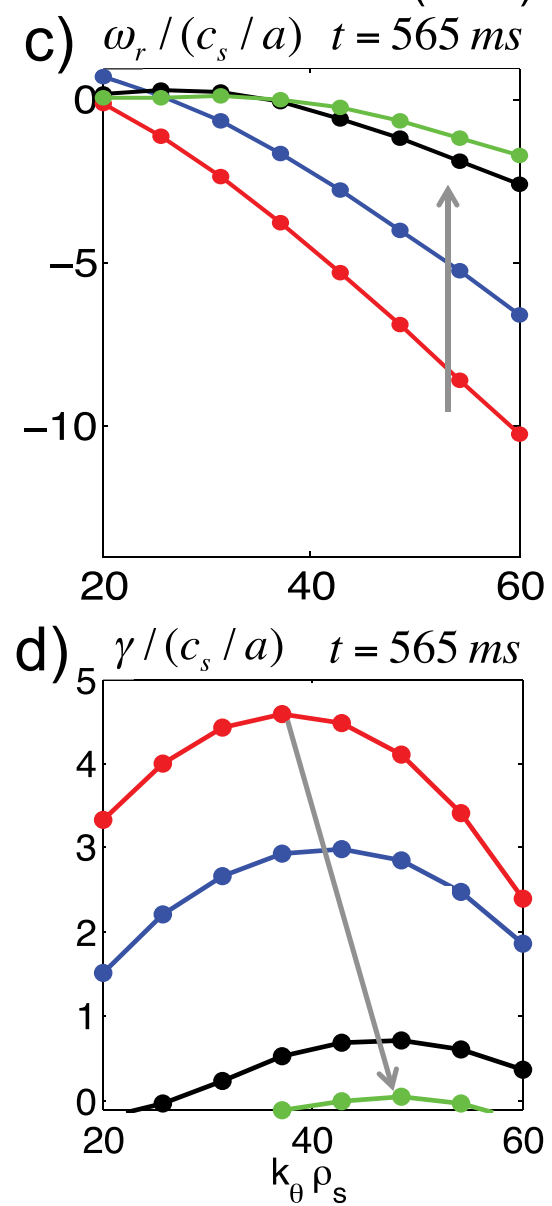

FIG. 10. A local scan on the electron density gradient is carried out at two times. At $t=398 \mathrm{~ms}$, the electron density gradient is low and subdominant in the critical gradient (Figs. 5(c) and 14(e)), and is shown to have negligible impact on the real frequency and linear growth rate. At $t=565 \mathrm{~ms}$, the electron density gradient is high and dominant in the critical gradient (Figs. 5(c) and 14(e)). At that time, a small change in the electron density gradient is shown to have a big impact on the real frequency and linear growth rate. 
frequency and linear growth rate at $t=565 \mathrm{~ms}$ computed with GS2 (Figs. 10(c) and 10(d)). In fact, additional scans carried out at different times (and not shown here) show that the more the electron density gradient is dominant, the bigger the observed effect on linear growth rate and real frequency. At $t=398 \mathrm{~ms}, R_{0} / L_{n_{e}}$ has a small value and is subdominant (cf. Figs. 5(c) and 14(e)). At $t=398 \mathrm{~ms}$, the real frequency and linear growth rate appear to be practically insensitive to small changes in $R_{0} / L_{n_{e}}$ (Figs. 10(a) and $10(\mathrm{~b}))$, contrasting with the high sensitivity at $t=565 \mathrm{~ms}$.

In Fig. 11 we make a very analogous analysis to that presented in Fig. 8, but this time using linear simulation results. A very clear parallel between Figs. 11 and 8 can be made if the wavenumber spectrum of fluctuations from experiment (Fig. 8(a)) is substituted with linear growth rates, as shown in Fig. 11(a). This can be argued by a simple mixing length argument (cf. Ref. 31). The Doppler subtracted plasma frame frequency of fluctuations from experiment is replaced by the real frequency from GS2.

Following the procedure established previously, at each time in the analysis (time span $0.348<t<0.565 \mathrm{~s}$ ), we calculate the maximum linear growth rate $\gamma_{\max } /\left(c_{s} / a\right)$ and the wavenumber corresponding to the maximum growth rate $k_{\theta} \rho_{s}\left(\gamma_{\max }\right)$ using linear gyrokinetic simulations with GS2. At each time, a local value of the electron density gradient is also calculated, and one can plot $\gamma_{\max } /\left(c_{s} / a\right)$ and $k_{\theta} \rho_{s}\left(\gamma_{\max }\right)$ as a function of the local value of the electron density gradient. The plots are shown in Figs. 11(c) and 11(d). At the particular time of $t=0.42 \mathrm{~s}$, these values are taken from the orange dot in Fig. 11(a) and mapped in Figs. 11(c) and 11(d). A very weak correlation is observed between $\gamma_{\max } /\left(c_{s} / a\right)$ and $R_{0} / L_{n_{e}}$. However, a clear correlation is observed between the wavenumber at maximum linear growth rate and the electron density gradient $R_{0} / L_{n_{e}}$, and the linear correlation coefficient is found to be $R^{2} \approx 85 \%$.

To exploit the correlation between real frequency and density gradient, we pick a fixed wavenumber, and calculate at each time the frequency corresponding to that fixed wavenumber. We choose the wavenumber $k_{\theta} \rho_{s}=30$, and the real frequency corresponding to that wavenumber for $t=0.42 \mathrm{~s}$ is shown in Fig. 11(b) as a red dot. In a similar way as was done with the linear growth rates, $\omega_{r}\left(k_{\theta} \rho_{s}=30\right)$ is plotted against the local value of $R_{0} / L_{n_{e}}$ at the corresponding time in Fig. 11(e) (see red dot corresponding to a time $t=0.42 \mathrm{~s}$ ). A very clear correlation is observed, with a correlation coefficient of $R^{2} \approx 91 \%$. This once more is a very clear indication, from the simulation, of the effect of the local density gradient on real frequency of instability calculated by GS2.

We conclude from this section stating that the local value of the electron density gradient has a weak effect on the high$\mathrm{k}$ linear growth rates (despite the high effect observed in the local scan in Fig. 10); however, it proves to be highly correlated with the wavenumber corresponding to the maximum linear growth rate and the real frequency of instability. It is important to note that the same trends from experimental data presented in Section II are reproduced using linear gyrokinetic simulation (e.g., compare Figs. 8 and 11).

\section{B. Preliminary nonlinear gyrokinetic simulation}

In this section we present preliminary results from nonlinear gyrokinetic simulations carried out using experimental parameters corresponding to the same NSTX discharge 141767 presented in Section II.

Local nonlinear ETG simulations were run using the Eulerian gyrokinetic code $\mathrm{GYRO}^{32}$ for two cases at $\mathrm{t}=398 \mathrm{~ms}(\mathrm{r} / \mathrm{a}=0.71)$ and $\mathrm{t}=565 \mathrm{~ms}(\mathrm{r} / \mathrm{a}=0.68)$ when the density gradient was lowest $\left(398 \mathrm{~ms}, R_{0} / L_{n_{e}} \approx 1.5\right)$ and highest $\left(565 \mathrm{~ms}, R_{0} / L_{n_{e}} \approx 6.5\right)$ at the scattering region (cf. Fig. 14(e)). The nonlinear simulations used MHD equilibrium reconstructions from LRDFIT $^{28}$ and plasma parameters derived from the measured experimental profiles including kinetic electrons and two kinetic ion species (D, C) with realistic mass ratios, collisions, shear, and compressional magnetic perturbations at finite- $\beta$ (although the transport is dominated by electrostatic perturbations), and $E \times B$ flow shear. The base nonlinear simulations used the following numerical grids as determined from previous studies and convergence tests: ${ }^{19,33}$ perpendicular box size $L_{x} \times L_{y} \approx 6 \times 4 \rho_{s}$

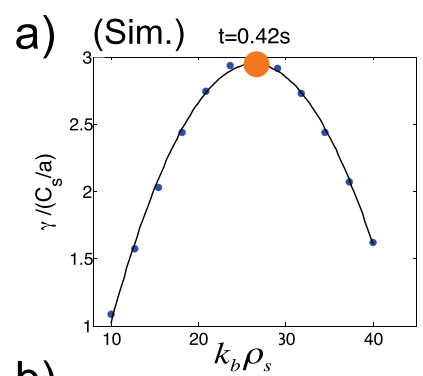

b)

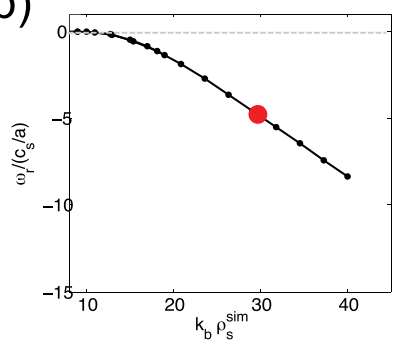

c)

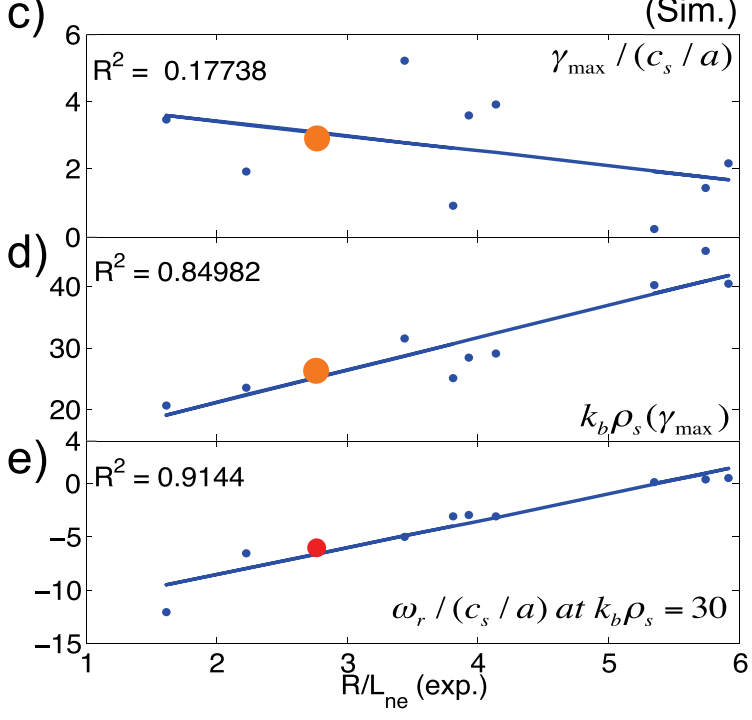

FIG. 11. At each time, the maximum linear growth rate $\gamma_{\max } /\left(c_{s} / a\right)$ and the wavenumber corresponding to the maximum linear growth rate $k_{\theta} \rho_{s}\left(\gamma_{\max }\right)$ are recorded (a), and are plotted against the local value of the density gradient in (c) and (d). To analyze the real frequency, the real frequency is calculated at a fixed wavenumber $\left(k_{\theta} \rho_{s}=30\right)$ and is plotted in (e) as a function of density gradient. 
$\left(360 \times 240 \rho_{e}\right), 48$ complex toroidal modes, 192 radial grid points, 12 energies, 12 pitch angles, and 14 parallel mesh points ( $\times 2$ signs of parallel velocity). We note that with the choice in box size, only electron scale turbulence is simulated $\left(k_{\theta} \rho_{s}^{\min }=1.5, k_{\theta} \rho_{s}^{\max }=73\right)$ with the assumption that ion scale turbulence is sufficiently suppressed at low $-k_{\theta} \rho_{s}$ by the $E \times B$ shear.

Figures 12(a) and 12(b) show the total electron heat flux (at the scattering location $R \approx 135 \mathrm{~cm}$ ) versus the normalized electron temperature gradient and the electron heat flux wavenumber spectrum, respectively. A scan on $R_{0} / L_{T_{e}}$ was carried out at $t=398 \mathrm{~ms}$ (low $R_{0} / L_{n_{e}}$ case, solid blue line) and $t=565 \mathrm{~ms}$ (high $R_{0} / L_{n_{e}}$ case, solid green line) to reproduce Fig. 12(a). The experimental cases (experimental $R_{0} / L_{T_{e}}$ ) are indicated by triangles, and the corresponding electron heat flux wavenumber spectrum is shown in Fig. 12(b). The low density gradient case at $t=398 \mathrm{~ms}$ gives rise to a much higher level of electron heat flux, a higher stiffness, and lower nonlinear threshold than the high density gradient case at $t=565 \mathrm{~ms}$. Note how the temperature gradient value is higher at $t=565 \mathrm{~ms}$ than at $t=398 \mathrm{~ms}$, suggesting a higher ETG-drive at $t=565 \mathrm{~ms}$. However, the predicted heat flux is orders of magnitude lower at $t=565 \mathrm{~ms}$, emphasizing the high impact of the density gradient on the predicted electron heat flux. At $t=398 \mathrm{~ms}$, power balance calculations provide an experimental level of electron heat flux $Q_{e} \sim 1.5 \mathrm{MW}$, about three times larger than the predicted $Q_{e}$ from GYRO (blue triangle). At $t=565 \mathrm{~ms}$, power balance calculations predict $Q_{e} \sim 0.9 \mathrm{MW}$, lower than at $t=398 \mathrm{~ms}$ but orders of magnitude higher than the predicted heat flux by GYRO (green triangle). This suggests ion-scale turbulence might have a substantial contribution to $Q_{e}$.

The green dashed line shows the result of an $R_{0} / L_{T_{e}}$ scan carried out using experimental parameters corresponding to the high density gradient case at $t=565 \mathrm{~ms}$, but using the density gradient value corresponding to the $t=398 \mathrm{~ms}$ case $\left(R_{0} / L_{n_{e}} \approx 1.5\right.$ instead of $\left.R_{0} / L_{n_{e}} \approx 6.5\right)$. This allows to explicitly separate the effect of the electron density gradient. In particular, the green open circle on the green dashed curve was calculated using the experimental value of $R_{0} / L_{T_{e}}$ (same as the green triangle). Note how the total electron heat flux is increased from a value of $Q_{e} \approx 0$ (green triangle) to a value of $Q_{e} \approx 1.6 \mathrm{MW}$ (green circle), a pure consequence of the density gradient. The stiffness is also highly increased (compare thick green and dashed green curves). The spectral shape of the electron heat flux is also recovered by simply changing the electron density gradient value (Fig. 12(b)). Note the electron heat flux for the dashed line is even higher than the $398 \mathrm{~ms}$ case. A possible explanation might be that the temperature gradient at $t=565 \mathrm{~ms}$ is higher than that at $t=398 \mathrm{~ms}$ (cf. red curve in Fig. 4(b)); however, this remains speculative. A careful look at the heat flux spectrum reveals how density gradient has preferentially stabilized lower- $k$ wavenumbers rather than higher$\mathrm{k}$, supporting the observation of density gradient stabilizing lower- $k$ high-k turbulence and shifting it to higher wavenumbers. Linear simulations seemed to support this observation (shift of wavenumber at peak linear growth rate with density gradient). Experimental observation is more complicated and difficult to interpret in the work presented here, although it was reported in Ref. 18.

In summary, the effect of the local value of the equilibrium density gradient on the predicted electron heat flux by local electron-scale nonlinear gyrokinetic simulations is evident, and reaffirms a nonlinear change in ETG transport and fluctuation spectrum from large equilibrium density gradient as predicted in previous ETG nonlinear simulations. ${ }^{19,34}$

\section{COMPARISONS BETWEEN EXPERIMENT AND GYROKINETIC SIMULATION}

In this section we present the experimental observations highlighting the effect of the density gradient as well as gyrokinetic simulations carried out for the corresponding NSTX plasma discharge. We show how the stabilizing effect of the density gradient is captured by the gyrokinetic simulations presented. We also show the similarities between the experimental and numerical results.
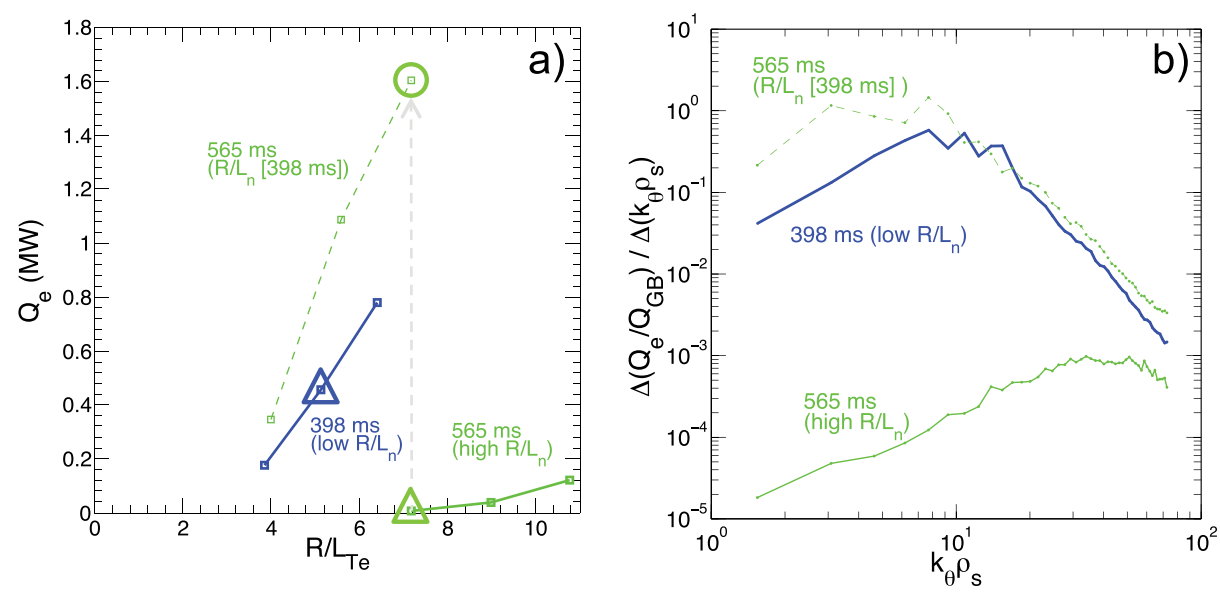

FIG. 12. (a) Predicted electron heat flux by GYRO as a function of $R_{0} / L_{T_{e}}$ for the high $R_{0} / L_{n_{e}}$ case (green) and low $R_{0} / L_{n_{e}}$ case (blue). Experimental cases are shown by blue and green triangles. The green dashed line shows the predicted heat flux at $\mathrm{t}=565 \mathrm{~ms}$, but using the density gradient value from $\mathrm{t}=398 \mathrm{~ms}$. The green circle is calculated by only varying the density gradient from $\mathrm{t}=565 \mathrm{~ms}$ (green triangle) to the value at $\mathrm{t}=398 \mathrm{~ms}\left(\right.$ low $R_{0} / L_{n_{e}}$ ). Note the consequent impact on $Q_{e}$. (b) Fractional electron heat flux spectrum corresponding to the experimental base cases (green and blue triangles), and at $\mathrm{t}=565 \mathrm{~ms}$ using the $R_{0} / L_{n_{e}}$ value from $\mathrm{t}=398 \mathrm{~ms}$ (corresponding to green circle in (a)). 


\section{A. Electron density gradient and real frequency}

In this subsection we show a comparison between the real frequency obtained from linear gyrokinetic simulations using GS2, and the Doppler subtracted plasma frame real frequency of fluctuations as detected by the high-k scattering diagnostic at NSTX.

In Figure 13 the plasma frame frequency of detected fluctuations is shown in (a), and the real frequency of instability is shown in (b). An important remark should be made here before the comparison is made. The experimental frequencies in (a) are plotted against the experimentally detected wavenumbers $k_{\perp} \rho_{s}^{\text {exp }}$ determined by ray tracing calculations. Detected wavenumbers by the high-k scattering system at NSTX are mostly radial, but have a small binormal component $k_{b}$, and $k_{r} \gg k_{b}$. In the present convention, $k_{\perp}=\sqrt{k_{r}^{2}+k_{b}^{2}}$. Recall that typical detected wavenumbers satisfy $k_{\perp} \sim 20 \mathrm{~cm}^{-1}$. Concerning the simulation wavenumbers in (b), they are all set to have a null radial component $k_{r}=0$. This condition corresponds to the most unstable mode of the instability. In that sense, the real frequencies in (b) are plotted against $k_{\theta} \rho_{s}^{s i m}$. The difference between the experimental and simulation wavenumbers precludes plotting the two curves together. In the discussion that follows we do not attempt to make quantitative comparisons between experiment and simulation and obtain agreement within errorbars. Our goal here is to look for a qualitative trend that might be present in both experiment and the simulation. One reasonable thing to do would be to run linear gyrokinetic simulations specifying the experimental value of the wavenumber as input, instead of setting $k_{r}=0$ from the start. One would need to perform an accurate mapping between the experimental $\left(k_{r}, k_{b}\right)$ pair (as computed using ray-tracing calculations) to a $\left(k_{r}, k_{\theta}\right)$ pair calculated according to GS2 geometry and used as input in the linear GS2 simulations. This would require a synthetic diagnostic tool and is object of future work. Nonlinear gyrokinetic simulations are also be needed to further explore the experimentally detected wavenumber.

We see in Figure 13 that the Doppler subtracted experimental frequency seems to increase in time from a lowest value at $t=398 \mathrm{~ms}$ to a highest value at $t=565 \mathrm{~ms}$. This same trend is observed from the linear simulations carried out, and presented in Fig. 13(b). In fact, from $t=398 \mathrm{~ms}$ to $t=565 \mathrm{~ms}$, the electron density gradient is increasing, as can be seen from Fig. 14(e). As the electron density gradient increases, the frequency is decreased (in absolute value) and approaches 0 . This is the same trend observed in the local density gradient scan in Section III (Fig. 10).

\section{B. Correlation between unstable wavenumbers and observed fluctuations}

It was shown in Section III how the electron density gradient is correlated to the wavenumber corresponding to the maximum linear growth rate as computed using linear GS2 gyrokinetic simulations. In this section we correlate the wavenumber at the maximum linear growth rate with the amplitude of the electron density fluctuations detected by the high-k scattering system.

In Fig. 14(a) are plotted the high-k and low-k linear growth rates. The dashed horizontal lines correspond to the $E \times B$ shearing rates computed using TRANSP calculations ${ }^{23}$ (using the Waltz-Miller definition ${ }^{24}$ ) at the analysis times. Similar levels between $E \times B$ shearing rates and low-k linear growth rates corresponding to the most unstable mode suggest that low-k (ITG) turbulence might be suppressed. In addition, TRANSP calculations show that the ion effective thermal diffusivity is at neoclassical levels (not shown here), consistent with the assumption that ion scale turbulence might be suppressed by $E \times B$ shear.

Linear growth rates (low-k and high-k) from Fig. 14(a) are not correlated with the total scattered power from the highk scattering diagnostic $\left(P_{t o t} \propto\left(\delta n_{e} / n_{e}\right)^{2}\right.$ in Fig. 14(d)). In fact, at $t=0.448 \mathrm{~s}$ the high-k linear growth rate attains the highest value among the four times in the analysis (see orange dot), but the fluctuation level in panel 14(d) is lowest at that time.

In order to reproduce Figs. 14(b) and 14(c), the maximum linear growth rate $\left(\gamma_{\max } /\left(c_{s} / a\right)\right)$ and the wavenumber corresponding to that maximum linear growth rate $k_{\theta} \rho_{s}\left(\gamma_{\max }\right)$ are calculated at each time in the analysis. These two quantities are then computed for several times, and are plotted in Figs. 14(b) and 14(c). From the orange dot in Fig. 14(a) at $t=0.448 \mathrm{~s}$, the values of $\gamma_{\max } /\left(c_{s} / a\right)$ and $k_{\theta} \rho_{s}\left(\gamma_{\max }\right)$ are retrieved and mapped in Figs. 14(b) and 14(c). No correlation is observed between $\gamma_{\max } /\left(c_{s} / a\right)$ and $P_{\text {tot }}$ (indicative of electron density fluctuations) from Fig. 14(d); however, a correlation can be established with the wavenumber at the
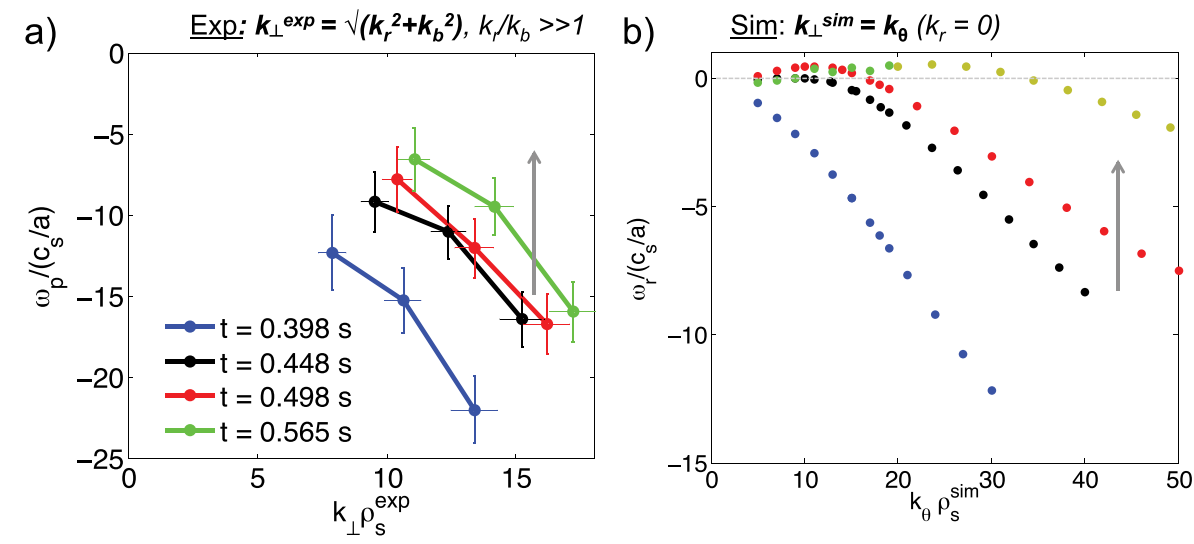

FIG. 13. (a) Doppler subtracted plasma frame frequency of fluctuations. (b) Real frequency of instability from GS2 linear gyrokinetic simulations. Note the very similar behavior of the real frequencies from experiment and simulation. 

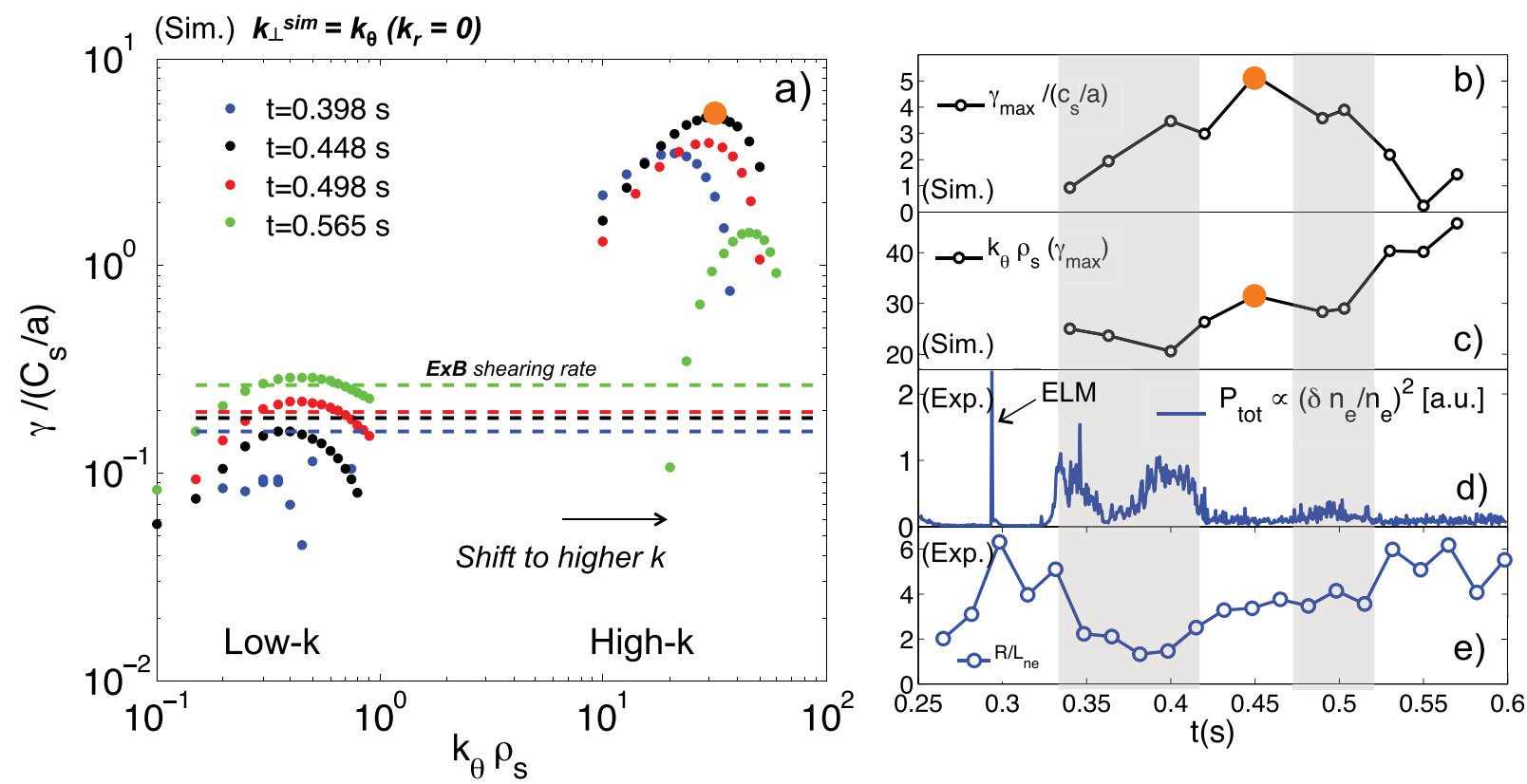

FIG. 14. (a) Linear growth rates computed with GS2 for low-k $\left(0.1 \lesssim k_{\theta} \rho_{s} \lesssim 1\right)$ and high-k wavenumbers $\left(10 \lesssim k_{\theta} \rho_{s} \lesssim 60\right)$. Notice the linearly unstable high$\mathrm{k}$ wavenumbers are shifting to even higher wavenumbers as time progresses. Horizontal lines correspond to $E \times B$ shearing rate from TRANSP calculations (Waltz-Miller definition ${ }^{24}$ ). (b) Maximum linear growth rate computed with GS2. (c) Wavenumber corresponding to the maximum growth rate computed with GS2. (d) Total scattered power (integrated in frequency) from channel 1 of the high-k scattering diagnostic. (e) Normalized electron density gradient $R_{0} / L_{n_{c}}$ computed at the scattering location. Notice the similarity between Figures 14(c) and 14(e).

maximum linear growth rate $k_{\theta} \rho_{s}\left(\gamma_{\max }\right)$. During the time $0.33 \lesssim t \lesssim 0.42 \mathrm{~s}$ (grey time panel), low $k_{\theta} \rho_{s}\left(\gamma_{\max }\right)$ corresponds to a high level of fluctuations. As $k_{\theta} \rho_{s}\left(\gamma_{\max }\right)$ increases between $0.4 \lessgtr t \leqq 0.45 \mathrm{~s}$, a clear reduction in density fluctuations is observed. A period of slight decrease of $k_{\theta} \rho_{s}\left(\gamma_{\max }\right)$ between $0.45 \lessgtr t \lesssim 0.5 \mathrm{~s}$ corresponds to a higher level of scattered power $P_{\text {tot }}$ (this last comment is a simple observation; it is not clear whether this small change in $k_{\theta} \rho_{s}\left(\gamma_{\max }\right)$ can have a significant impact on density fluctuations and turbulence). A clear increase in $k_{\theta} \rho_{s}\left(\gamma_{\max }\right)$ for $0.5 \lessgtr t \lesssim 0.57 \mathrm{~s}$ agrees with a further reduced level of fluctuations.

One additional correlation might be established with the experimental value of the electron density gradient. A similar shape in the curve $k_{\theta} \rho_{s}\left(\gamma_{\max }\right)$ in time (Fig. 14(c)) and the local value of $R_{0} / L_{n_{e}}$ (Fig. 14(e)) suggests a clear correlation. An analysis of these two curves provides an $R^{2}$ linear correlation coefficient between $k_{\theta} \rho_{s}\left(\gamma_{\max }\right)$ and $R_{0} / L_{n_{e}}$ of $R^{2} \approx 85 \%$ (already shown in Fig. 11(d); note here $R^{2}$ denotes a linear correlation coefficient and not major radius). The previous correlation between fluctuations $\left(P_{t o t}\right.$ in Fig. 14(d)) and $k_{\theta} \rho_{s}\left(\gamma_{\max }\right)$ (Fig. 14(c)) can be extended to include the local electron density gradient $R_{0} / L_{n_{e}}$ (Fig. 14(e)).

The previous comments allow us to argue that the electron density gradient might be driving high-k turbulence levels (Fig. 14(d)) to even higher wavenumbers, and at the same time stabilizing lower- $k$ high-k turbulence. The fluctuation level in Fig. 14(d) is indicative of high-k turbulence, and it seems to be driven to even higher wavenumbers by the effect of the local electron density gradient.

Linear simulation and nonlinear simulations qualitatively agree on the influence of the electron density gradient (Fig. 15): high density gradient appears to reduce the lower-k part of the linear growth rate and electron heat flux spectrum
(Figs. 15(b) and 15(c), respectively), and at the same time shifting the spectrum to higher wavenumbers. Experiment also suggests that the lower- $k$ part of the high-k fluctuation spectrum $\left(\delta n_{e} / n_{e}\right)^{2}$ might be reduced due to density gradient (Fig. 15(a)), but a definite statement should not be made at this stage owing to the measurement limitations of the high$\mathrm{k}$ scattering system and refraction effects of density gradient on the probe beam. Despite, a qualitative connection can be established among experiment, linear, and nonlinear simulations, which all support the experimental observation of density gradient stabilization of high-k turbulence.

\section{CONCLUSION AND DISCUSSION}

We have reported here on the influence of the local value of the normalized electron density gradient on experimental electron density fluctuations measured by the high- $\mathrm{k}$ scattering system at NSTX. We observe that the linear threshold determined by critical gradient expression (Eq. (1)) determines presence of high-k fluctuations (Fig. 4). No fluctuations are observed when the local electron temperature gradient is at marginal stability levels with the critical temperature gradient $\left(R / L_{T_{e}}^{e x p} \sim\left(R / L_{T_{e}}\right)_{\text {crit }}\right)$, and electron density fluctuations are detected during unstable periods when $R / L_{T_{e}}^{\text {exp }}>\left(R / L_{T_{e}}\right)_{c r i t}$. The local value of the density gradient seems to be a main actor affecting high-k turbulence levels in the plasma discharge presented here. The electron density gradient is the dominant term in the critical gradient expression (1) for most part of the discharge, and this might be an explanation for the large influence of the electron density gradient in this plasma. The electron density gradient is also observed to be a nonlinear stabilizing mechanism on high-k turbulence: for similar values of the difference 

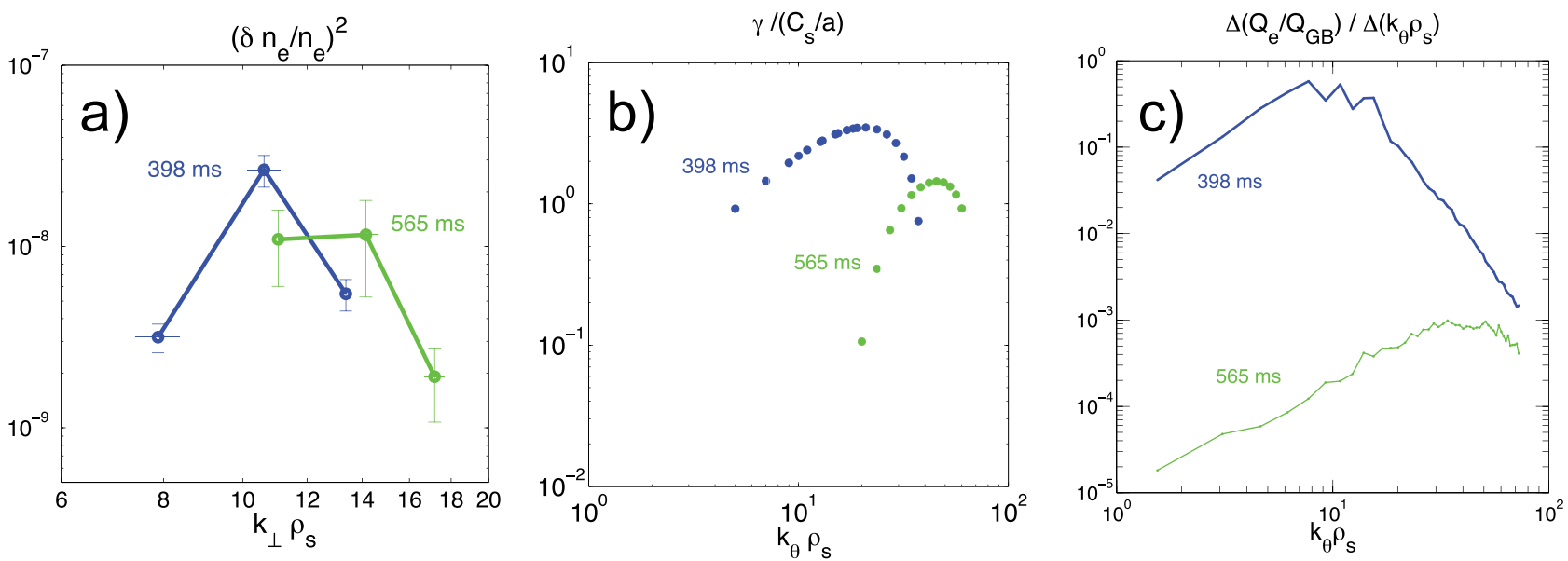

FIG. 15. (a) Experimental amplitude of electron density fluctuations from the high-k scattering diagnostic. (b) Linear growth rates predicted by GS2 linear simulations. (c) Fractional electron heat flux spectrum predicted by GYRO electron scale nonlinear simulations. In blue are results carried out for the low $R_{0} / L_{n_{e}}$ case and in green for the high $R_{0} / L_{n_{e}}$ case. The similar behavior between the three figures from experiment, linear simulation, and nonlinear simulation establishes a qualitative connection between simulation and experiment, and reinforces the experimental observation of density gradient stabilization of high- $\mathrm{k}$ turbulence.

$R / L_{T_{e}}^{\text {exp }}-\left(R / L_{T_{e}}\right)_{c r i t}$, low electron density gradient is correlated with high levels of electron density fluctuations and vice-versa (Fig. 5). In addition, the Doppler subtracted plasma frame frequency of fluctuations appears to decrease with increasing electron density gradient (Figs. 13 and 11).

Linear gyrokinetic simulations do not show a clear correlation between the electron density gradient and the amplitude of the linear growth rates (Figs. 14 and 11) despite the high impact shown in the density gradient scan (Fig. 10). It may be that density gradient indeed has a profound effect on linear growth rates when it is dominant in the critical gradient, but this correlation might be hidden in Figs. 14 and 11 due to the change of other plasma parameters that might equally affect the value of linear growth rates. Thus we do not claim here a direct correlation between the amplitude of the linear growth rate and density gradient. However, the wavenumber corresponding to the maximum linear growth rate is shown to have a clear correlation with density gradient (Figs. 14, 10, and 11). The real frequency from GS2 linear runs is shown to have a very strong dependence on the local value of the electron density gradient, and exhibits the same behavior as the Doppler-subtracted plasma-frame frequency of fluctuations (from experiment). A local scan on $R_{0} / L_{n_{e}}$ carried out with GS2 confirms the previous statements (Fig. 10), and the same trends are found between experiments and linear gyrokinetic simulations as shown in Figs. 8 and 11.

Nonlinear gyrokinetic simulations were carried out at times when the density gradient was highest and lowest, and show that the electron heat flux is much higher for the low density gradient case $(t=398 \mathrm{~ms})$ than for the high density gradient case $(t=565 \mathrm{~ms}$, Fig. 12). The electron heat flux is also greatly increased when a density gradient scan was performed on the high density gradient case to match the low density gradient.

Several interesting questions remain that will be explored in future work. The electron scale nonlinear gyrokinetic simulations presented in Section III assume that low-k ion scale turbulence is suppressed by $E \times B$ shear. This is indeed a reasonable assumption: the $E \times B$ shearing rate is shown to be at the same level as low-k linear growth rates of instability (Fig. 14) and the ion thermal diffusivity predicted by power balance calculations (TRANSP) is at neoclassical levels at $t=565 \mathrm{~ms}$ (not shown here), both suggesting that low-k turbulence might be suppressed by $E \times B$ shear. The electron heat flux predicted for the high density gradient case $(t=565 \mathrm{~ms})$ has a very low value (Fig. 12). In fact, the experimental base case (green triangle) predicts practically no electron heat flux. This abnormally low value is unlikely to account for experimental transport levels. The convergence of this simulation was tested by running an additional simulation where the value of $k_{\theta} \rho_{s}^{\max }$ was doubled (from 73 to 146 , not shown here). Both simulations predict the same level of $Q_{e}$. One is tempted to assume that ion scale turbulence might have a contribution to $Q_{e}$, which could account for experimental levels of electron heat flux. However, this would contradict the previous assumption of low-k turbulence suppression by $E \times B$ shear. The contribution of low-k ion scale turbulence is unclear in this case, and remains subject of future work. Nonlinear gyrokinetic simulations also show a nonlinear upshift of the critical gradient (Fig. 12) with respect to the linear critical gradient (Fig. 5) calculated using the critical gradient formula and GS2 linear scans. Further investigation of this phenomenon is the subject of future work.

Local, and possibly global, ion-scale nonlinear simulations might be needed to correctly assess the contribution of ion scale turbulence to electron thermal transport, and ultimately multi-scale simulations might be necessary to correctly discern the contributions from high-k and low-k turbulence on electron thermal transport. This would allow to establish more quantitative comparisons between experiment and simulation, and are subject of future investigation.

\section{ACKNOWLEDGMENTS}

The author would like to thank all the coauthors and the NSTX Team. The author would particularly like to thank N. A. Crocker for enlightening discussions. This work has been 
supported by US. D.O.E. Contract No. DE-AC0209CH11466. Computer simulations were carried out at the National Energy Research Scientific Computing Center, supported by the Office of Science of the U.S. Department of Energy under Contract No. DE-AC02-05CH11231. The digital data for this paper can be found in http:// arks.princeton.edu/ark:/88435/dsp018p58pg29j.

${ }^{1}$ D. R. Smith, E. Mazzucato, W. Lee, H. K. Park, C. W. Domier, and N. C. Luhmann, Jr., Rev. Sci. Instrum. 79, 123501 (2008).

${ }^{2}$ F. Jenko, W. Dorland, and G. W. Hammett, Phys. Plasmas 8, 4096 (2001). ${ }^{3}$ M. Kotschenreuther, G. Rewoldt, and W. M. Tang, Comput. Phys. Commun. 88, 128 (1995).

${ }^{4}$ J. E. Menard, L. Bromberg, T. Brown, T. Burgess, D. Dix, L. El-Guebaly, T. Gerrity, R. J. Goldston, R. J. Hawryluk, R. Kastner, C. Kessel, S. Malang, J. Minervini, G. H. Neilson, C. L. Neumeyer, S. Prager, M. Sawan, J. Sheffield, A. Sternlieb, L. Waganer, D. Whyte, and M. Zarnstorff, Nucl. Fusion 51, 103014 (2011).

${ }^{5}$ W. Horton, Rev. Mod. Phys. 71, 735 (1999)

${ }^{6} \mathrm{~W}$. Horton, Turbulent Transport in Magnetized Plasmas (World Scientific, London, 2012).

${ }^{7}$ W. Horton, H. V. Wong, P. J. Morrison, A. Wurm, J. H. Kim, J. C. Perez, J. Pratt, G. T. Hoang, B. P. LeBlanc, and R. Ball, Nucl. Fusion 45, 976-985 (2005).

${ }^{8}$ J. W. Connor and H. R. Wilson, Plasma Phys. Controlled Fusion 36, 719 (1994).

${ }^{9}$ W. Dorland, F. Jenko, M. Kotschenreuther, and B. N. Rogers, Phys. Rev. Lett. 85, 5579 (2000).

${ }^{10}$ F. Jenko, W. Dorland, M. Kotschenreuther, and B. N. Rogers, Phys. Plasmas 7, 1904 (2000).

${ }^{11}$ F. Jenko and W. Dorland, Phys. Rev. Lett. 89, 225001 (2002).

${ }^{12}$ R. E. Waltz, J. Candy, and M. Fahey, Phys. Plasmas 14, 056116 (2007).

${ }^{13}$ S. M. Kaye, F. M. Levinton, D. Stutman, K. Tritz, H. Yuh, M. G. Bell, R. E. Bell, C. W. Domier, D. Gates, W. Horton, J. Kim, B. P. LeBlanc, N. C. Luhmann, Jr., R. Maingi, E. Mazzucato, J. E. Menard, D. Mikkelsen, D. Mueller, H. Park, G. Rewoldt, S. A. Sabbagh, D. R. Smith, and W. Wang, Nucl. Fusion 47, 499 (2007).

${ }^{14}$ D. R. Smith, S. M. Kaye, W. Lee, E. Mazzucato, H. K. Park, R. E. Bell, C. W. Domier, B. P. LeBlanc, F. M. Levinton, N. C. Luhmann, Jr., J. E. Menard, and H. Yuh, Phys. Rev. Lett. 102, 225005 (2009).
${ }^{15}$ Y. Ren, W. Guttenfelder, S. M. Kaye, E. Mazzucato, R. E. Bell, A. Diallo, C. W. Domier, B. P. LeBlanc, K. C. Lee, M. Podesta, D. R. Smith, and H. Yuh, Nucl. Fusion 53, 083007 (2013).

${ }^{16}$ H. Y. Yuh, F. M. Levinton, R. E. Bell, J. C. Hosea, S. M. Kaye, B. P. LeBlanc, E. Mazzucato, J. L. Peterson, D. R. Smith, J. Candy, R. E. Waltz, C. W. Domier, N. C. Luhmann, Jr., W. Lee, and H. K. Park, Phys. Plasmas 16, 056120 (2009).

${ }^{17}$ H. Y. Yuh, S. M. Kaye, F. M. Levinton, E. Mazzucato, D. R. Mikkelsen, D. R. Smith, R. E. Bell, J. C. Hosea, B. P. LeBlanc, J. L. Peterson, H. K. Park, and W. Lee, Phys. Rev. Lett. 106, 055003 (2011).

${ }^{18}$ Y. Ren, S. M. Kaye, E. Mazzucato, W. Guttenfelder, R. E. Bell, C. W. Domier, B. P. LeBlanc, K. C. Lee, N. C. Luhmann, Jr., D. R. Smith, and H. Yuh, Phys. Rev. Lett. 106, 165005 (2011).

${ }^{19}$ Y. Ren, W. Guttenfelder, S. M. Kaye, E. Mazzucato, R. E. Bell, A. Diallo, C. W. Domier, B. P. LeBlanc, K. C. Lee, D. R. Smith, and H. Yuh, Phys. Plasmas 19, 056125 (2012).

${ }^{20}$ E. Mazzucato, Phys. Plasmas 10, 753 (2003).

${ }^{21}$ E. Mazzucato, Plasma Phys. Controlled Fusion 48, 1749 (2006).

${ }^{22}$ S. M. Kaye, W. Solomon, R. E. Bell, B. P. LeBlanc, F. Levinton, J. Menard, G. Rewoldt, S. Sabbagh, W. Wang, and H. Yuh, Nucl. Fusion 49, 045010 (2009).

${ }^{23}$ R. J. Hawryluk, Physics of Plasma Close to Thermonuclear Conditions (Pergamon, New York, 1981).

${ }^{24}$ R. E. Waltz and R. L. Miller, Phys. Plasmas 6, 4265 (1999).

${ }^{25}$ E. Mazzucato, D. R. Smith, R. E. Bell, S. M. Kaye, J. C. Hosea, B. P. LeBlanc, J. R. Wilson, P. M. Ryan, C. W. Domier, N. C. Luhmann, Jr., H. Yuh, W. Lee, and H. Park, Phys. Rev. Lett. 101, 075001 (2008).

${ }^{26}$ B. P. LeBlanc, R. E. Bell, D. W. Johnson, D. E. Hoffman, D. C. Long, and R. W. Palladino, Rev. Sci. Instrum. 74, 1659 (2003).

${ }^{27}$ R. E. Bell, Rev. Sci. Instrum. 77, 10E902 (2006).

${ }^{28}$ J. E. Menard, R. E. Bell, D. A. Gates, S. M. Kaye, B. P. LeBlanc, F. M. Levinton, S. S. Medley, S. A. Sabbagh, D. Stutman, K. Tritz, and H. Yuh, Phys. Rev. Lett. 97, 095002 (2006).

${ }^{29}$ F. M. Levinton and H. Yuh, Rev. Sci. Instrum. 79, 10F522 (2008).

${ }^{30}$ R. L. Miller, M. S. Chu, J. M. Greene, Y. R. Lin-Liu, and R. E. Waltz, Phys. Plasmas 5, 973 (1998).

${ }^{31}$ X. Garbet, Plasma Phys. Controlled Fusion 43, A251-A266 (2001).

${ }^{32}$ J. Candy and R. E. Waltz, Phys. Rev. Lett. 91, 045001 (2003).

${ }^{33}$ W. Guttenfelder and J. Candy, Phys. Plasmas 18, 022506 (2011).

${ }^{34}$ W. Guttenfelder, J. L. Peterson, J. Candy, S. M. Kaye, Y. Ren, R. E. Bell, G. W. Hammett, B. P. LeBlanc, D. R. Mikkelsen, W. M. Nevins, and H. Yuh, Nucl. Fusion 53, 093022 (2013). 